\title{
Low-Reynolds-number hydrodynamic interactions in a suspension of spherical particles with slip surfaces
}

\author{
Huan J. Keh* and Shih H. Chen ${ }^{\dagger}$ \\ Department of Chemical Engineering, National Taiwan University, Taipei 106-17, Taiwan, \\ Republic of China
}

(Received 11 March 1996)

\begin{abstract}
The motion of two rigid spherical particles in an arbitrary configuration in an infinite viscous fluid at low Reynolds numbers is considered. The fluid is allowed to slip at the surfaces of the spheres and the particles may differ in radius. The resistance and mobility functions that completely characterize the linear relations between the forces and torques and the translational and rotational velocities of the particles are analytically calculated in the quasi-steady limit using a method of twin multipole expansions. For each function, an expression of power series in $r^{-1}$ is obtained, where $r$ is the distance between the particle centers. The agreement between these expressions and the relevant results in the literature is quite good. Based on a microscopic model, the analytical results for two-sphere hydrodynamic interactions are used to find the effect of the volume fraction of particles of each type on the average settling velocities in a bounded suspension of slip spheres. Our results, presented in simple closed forms, agree very well with the existing solutions for the limiting cases of no slip and perfect slip at the particles surfaces. In general, the particle-interaction effects are found to be more significant when the slip coefficients at the particle surfaces become smaller. Also, the influence of the interactions on the smaller particles is stronger than on the larger ones. (C) 1997 Elsevier Science Ltd. All rights reserved
\end{abstract}

Keywords: Two-particle hydrodynamic interactions; effects of volume fraction; slip spherical particles.

\section{INTRODUCTION}

The area of the moving of solid particles or fluid drops in a continuous medium at very small Reynolds numbers has continued to receive much attention from investigators in the fields of chemical, biomedical and environmental engineering and science. The majority of the moving phenomena are fundamental in nature, but permit one to develop rational understanding of many practical systems and industrial processes such as sedimentation, floatation, coagulation, spray drying, aerosol processing and motion of blood cells in an artery or vein. The theoretical study of this subject has grown out of the classic work of Stokes (1851) for a translating rigid sphere in a viscous fluid. Hadamard (1911) and Rybczynski (1911) have independently extended this result to the translation of a fluid sphere. Assuming continuous velocity and continuous tangential stress across the interface of fluid phases, they found that the force exerted on a spherical drop

*Corresponding author.

${ }^{\dagger}$ Present address: Department of Chemical Engineering, Hwa Hsia College, Taipei 235, Taiwan. of radius $a$ by the surrounding fluid of viscosity $\eta$ is given by

$$
\mathbf{F}^{(0)}=-6 \pi \eta a \frac{3 \eta^{*}+2}{3 \eta^{*}+3} \mathbf{U}
$$

where $\mathbf{U}$ is the migration velocity of the drop and $\eta^{*}$ is the internal-to-external viscosity ratio. Since the fluid viscosities are arbitrary, eq. (1) degenerates to the case of translation of a solid sphere (Stokes' law) when $\eta^{*} \rightarrow \infty$ and to the case of motion of a gas bubble with spherical shape in the limit $\eta^{*} \rightarrow 0$.

In most practical applications, multiparticle systems are more important than the single-particle situation; the latter condition can represent only the limiting case at low dispersed phase hold-up. In dispersions, particle interactions can be of primary importance and are related to the concentration dependence of the ensemble-averaged settling velocities of the particles (Batchelor, 1972; Reed and Anderson, 1980) and of the effective transport properties (Jeffrey, 1973; Batchelor, 1976, 1977). Problems concerning the hydrodynamic interactions between two or more fluid particles with arbitrary values of $\eta^{*}$ have been treated extensively in the past. Summaries for the current 
state of knowledge in this area and some informative references can be found in Kim and Karrila (1991) and Keh and Tseng (1992).

When one tries to solve the Navier-Stokes equations, it is usually assumed that no slippage arises at the solid-fluid interfaces. Actually, this is an idealization of occurrence of the transport processes. The phenomenon that the adjacent fluid (especially if the fluid is a slightly rarefied gas) can slip over a solid surface has been confirmed, both experimentally and theoretically (Kennard, 1938; Loyalka, 1990; Ying and Peters, 1991; Hutchins et al., 1995). Presumably, any such slipping would be proportional to the local tangential stress next to the solid surface (Basset, 1961; Happel and Brenner, 1983), at least as long as the velocity gradient is small. The constant of proportionality, $\beta^{-1}$, may be termed a 'slip coefficient'. The quantity $\eta / \beta$ is a length, which can be pictured by noting that the fluid motion is the same as if the solid surface was displaced inward by a distance $\eta / \beta$ with the velocity gradient extending uniformly right up to no-slip velocity at the surface. Basset (1961) has found that the drag force acting on a translating rigid sphere with a slip-flow boundary condition at its surface (e.g. a settling aerosol sphere) is

$$
\mathbf{F}^{(0)}=-6 \pi \eta a \frac{\beta \mathrm{a}+2 \eta}{\beta \mathrm{a}+3 \eta} \mathbf{U} .
$$

When $\beta \rightarrow \infty$, there is no slip at the particle surface and eq. (2) degenerates to Stokes' law. In the limiting case of $\beta=0$, there is a perfect slip at the particle surface (the particle acts like a spherical gas bubble) and eq. (2) is consistent with eq. (1) (taking $\eta^{*}=0$ ). Note that, as can be seen from eqs (1) and (2), the flow field produced by the migration of a 'slip' solid sphere is the same as the external flow field generated by the same motion of a fluid drop with a value of $\eta^{*}$ equal to $\beta a / 3 \eta$.

In eq. (2), the slip coefficient has been determined experimentally for various cases and found to agree with the general kinetic theory of gases. It can be evaluated from the relation

$$
\beta^{-1}=C_{m} l / \eta
$$

where $l$ is the mean free path of a gas molecule and $C_{m}$ is a dimensionless constant related to the momentum accommodation coefficient at the solid surface. Although $C_{m}$ surely depends upon the nature of the surface, examination of the experimental data suggests that it will be in the range 1.0-1.5 (Davis, 1972; Talbot et al., 1980; Loyalka, 1990). Note that the slip-flow boundary condition is not only applicable for a gas-solid surface in the continuum regime (Knudsen number $l / a \ll 1$ ), but also appears to be valid for some cases even into the molecular flow regime $(l / a \geqslant 1)$.

The hydrodynamic interactions between two solid particles with finite values of $\beta a / \eta$ are different, both physically and mathematically, from those between two fluid drops of finite viscosities. Through an exact representation in spherical bipolar coordinates, the creeping motion of two rigid spheres with slip surfaces translating along the line of their centers was examined by Reed and Morrison (1974) and Chen and Keh (1995). Numerical results to correct Basset's equation (2) for each particle were presented for various cases. It was found that the interaction effect between particles decreases with the increase of the slip coefficients at the particle surfaces. This interaction effect can be very significant when the distance between particle surfaces approaches zero. The influence of the interactions between particles, in general, is far greater on the smaller one than on the larger one.

The objective of the present work is to study the hydrodynamic interactions analytically, between two slip spherical particles in a general situation. The particles may differ in radius and have arbitrary translational and rotational velocities (or applied forces and torques). A method of twin multipole expansions (Jeffrey and Onishi, 1984) is used to solve the problem. The detailed discussion of the hydrodynamic interactions between two rigid spheres is presented in Section 2, where we review the relevant resistance and mobility functions. In Section 3, the formulation of the method of twin multipole expansions for the arbitrary motion of two slip spheres is given. In Sections 4 and 5 , the asymptotic formulas for the resistance and mobility functions, respectively, expressed in terms of the dimensionless slip coefficients, the relative separation distance and the size ratio of the two spherical particles are derived. Our results agree well with the existing solutions for the interactions between two spheres in the literature. Finally, in Section 6 , the results of two-sphere interactions obtained in Section 5 are employed to evaluate the effect of the volume fraction of particles on the average settling velocity in a bounded suspension of small slip spheres, and the general result is obtained in eqs (92) and (93). For the special cases of a suspension of no-slip spheres and of perfect-slip spheres (gas bubbles), analytical expressions for the average settling velocity with high accuracy are given in eqs (94) and (95), respectively.

\section{DEFINITION OF THE INTERACTIONS BETWEEN TWO RIGID SPHERES}

In this work we use the definition of two-sphere hydrodynamic interactions summarized by Jeffrey and Onishi (1984). Two rigid spheres are immersed in an unbounded fluid with the undisturbed velocity field $\mathbf{V}(\mathbf{x})=\mathbf{V}_{0}+\boldsymbol{\omega} \times \mathbf{x}$. Sphere $\alpha$ has radius $a_{\alpha}$, angular velocity $\boldsymbol{\Omega}_{\alpha}$, and translational velocity $\mathbf{U}_{\alpha}$ at its center $\mathbf{x}_{\alpha}$, where $\alpha=1$ or 2 . The force and torque exerted by sphere $\alpha$ on the fluid about the center of the sphere are $\mathbf{F}_{\alpha}$ and $\mathbf{T}_{\alpha}$, respectively. The relations between the quantities $\mathbf{U}_{\alpha}, \boldsymbol{\Omega}_{\alpha}, \mathbf{F}_{\alpha}, \mathbf{T}_{\alpha}, \mathbf{V}_{0}$ and $\boldsymbol{\omega}$ are the interactions to be determined.

\subsection{The resistance problem}

When the specified quantities are the velocities of the particles and of the prescribed flow, the linearity of the Stokes equations [given in eq. (15)] permits the 
expression of the forces and torques in the form

$$
\begin{aligned}
{\left[\begin{array}{c}
\mathbf{F}_{1} \\
\mathbf{F}_{2} \\
\mathbf{T}_{1} \\
\mathbf{T}_{2}
\end{array}\right]=} & {\left[\begin{array}{llll}
\mathbf{A}^{11} & \mathbf{A}^{12} & \tilde{\mathbf{B}}^{11} & \tilde{\mathbf{B}}^{12} \\
\mathbf{A}^{21} & \mathbf{A}^{22} & \tilde{\mathbf{B}}^{21} & \tilde{\mathbf{B}}^{22} \\
\mathbf{B}^{11} & \mathbf{B}^{12} & \mathbf{C}^{11} & \mathbf{C}^{12} \\
\mathbf{B}^{21} & \mathbf{B}^{22} & \mathbf{C}^{21} & \mathbf{C}^{22}
\end{array}\right] } \\
& {\left[\begin{array}{c}
\mathrm{U}_{1}-\mathbf{V}\left(\mathbf{x}_{1}\right) \\
\mathbf{U}_{2}-\mathbf{V}\left(\mathbf{x}_{2}\right) \\
\mathbf{\Omega}_{1}-\omega \\
\mathbf{\Omega}_{2}-\omega
\end{array}\right] . }
\end{aligned}
$$

The square matrix of second-rank tensors in the above equation is the resistance matrix. The reciprocal theorem of Lorentz (Happel and Brenner, 1983) shows that the resistance matrix is symmetric, i.e.

$$
A_{i j}^{\alpha \beta}=A_{j i}^{\beta \alpha}, \quad \tilde{B}_{i j}^{\alpha \beta}=B_{j i}^{\beta \alpha}, \quad C_{i j}^{\alpha \beta}=C_{j i}^{\beta \alpha} .
$$

The two-sphere geometry implies that any element tensor $\mathbf{P}$ of the resistance matrix obeys

$$
\mathbf{P}^{\alpha \beta}\left(\mathbf{r}, a_{1}, a_{2}\right)=\mathbf{P}^{(3-\alpha)(3-\beta)}\left(-\mathbf{r}, a_{2}, a_{1}\right)
$$

where $\mathbf{r}=\mathbf{x}_{2}-\mathbf{x}_{1}=r \mathbf{e}(r=|\mathbf{r}|$ and $\mathbf{e}$ is the unit vector along $\mathbf{r}$ ) is the vector from the center of particle 1 to the center of particle 2 . Finally, the axial symmetry about $\mathbf{r}$ implies that each tensor in the resistance matrix can be reduced to an expression involving no more than two scalar functions. Thus, one can write

$$
\begin{aligned}
& \mathbf{A}^{\alpha \beta}=X_{\alpha \beta}^{A} \mathbf{e e}+Y_{\alpha \beta}^{A}(\mathbf{I}-\mathbf{e e}) \\
& \mathbf{B}^{\alpha \beta}=-\tilde{\mathbf{B}}^{\beta \alpha}=Y_{\alpha \beta}^{B} \mathbf{e} \times \mathbf{I} \\
& \mathbf{C}^{\alpha \beta}=X_{\alpha \beta}^{C} \mathbf{e e}+Y_{\alpha \beta}^{C}(\mathbf{I}-\mathbf{e e})
\end{aligned}
$$

where $I$ is the unit dyadic.

We now non-dimensionalize these tensors and their scalar functions so that they become functions only of two dimensionless variables

$$
s=\frac{2 r}{a_{1}+a_{2}} \quad \text { and } \quad \lambda=\frac{a_{2}}{a_{1}} .
$$

The non-dimensional resistance tensors are indicated by a caret with the definition

$$
\begin{aligned}
& \mathbf{A}^{\alpha \beta}=3 \pi\left(a_{\alpha}+a_{\beta}\right) \hat{\mathbf{A}}^{\alpha \beta} \\
& \mathbf{B}^{\alpha \beta}=\pi\left(a_{\alpha}+a_{\beta}\right)^{2} \hat{\mathbf{B}}^{\alpha \beta} \\
& \mathbf{C}^{\alpha \beta}=\pi\left(a_{\alpha}+a_{\beta}\right)^{3} \hat{\mathbf{C}}^{\alpha \beta} .
\end{aligned}
$$

Combining eqs (5)-(9), one can show that

$$
\begin{aligned}
& \hat{X}_{\alpha \beta}^{A}(s, \lambda)=\hat{X}_{\beta \alpha}^{A}(s, \lambda)=\hat{X}_{(3-\alpha)(3-\beta)}^{A}\left(s, \lambda^{-1}\right) \\
& \hat{Y}_{\alpha \beta}^{A}(s, \lambda)=\hat{Y}_{\beta \alpha}^{A}(s, \lambda)=\hat{Y}_{(3-\alpha)(3-\beta)}^{A}\left(s, \lambda^{-1}\right) \\
& \hat{Y}_{\alpha \beta}^{B}(s, \lambda)=-\hat{Y}_{(3-\alpha)(3-\beta)}^{B}\left(s, \lambda^{-1}\right) \\
& \hat{X}_{\alpha \beta}^{C}(s, \lambda)=\hat{X}_{\beta \alpha}^{C}(s, \lambda)=\hat{X}_{(3-\alpha)(3-\beta)}^{C}\left(s, \lambda^{-1}\right) \\
& \hat{Y}_{\alpha \beta}^{C}(s, \lambda)=\hat{Y}_{\beta \alpha}^{C}(s, \lambda)=\hat{Y}_{(3-\alpha)(3-\beta)}^{C}\left(s, \lambda^{-1}\right) .
\end{aligned}
$$

Thus, there are 10 independent non-dimensional scalar resistance functions to be determined for $2 \leqslant s$ $<\infty$ and $0 \leqslant \lambda<\infty$.

\subsection{The mobility problem}

When the particle forces and torques are prescribed in the ambient velocity field, one can write

$\left[\begin{array}{c}\mathbf{U}_{1}-\mathbf{V}\left(\mathbf{x}_{1}\right) \\ \mathbf{U}_{2}-\mathbf{V}\left(\mathbf{x}_{2}\right) \\ \mathbf{\Omega}_{1}-\omega \\ \mathbf{\Omega}_{2}-\omega\end{array}\right]=\eta^{-1}$

$$
\times\left[\begin{array}{llll}
a^{11} & a^{12} & \tilde{b}^{11} & \tilde{b}^{12} \\
a^{21} & a^{22} & \tilde{b}^{21} & \tilde{b}^{22} \\
b^{11} & b^{12} & c^{11} & c^{12} \\
b^{21} & b^{22} & c^{21} & c^{22}
\end{array}\right]\left[\begin{array}{l}
F_{1} \\
F_{2} \\
T_{1} \\
T_{2}
\end{array}\right]
$$

where the square matrix is the mobility matrix. The reciprocal theorem shows that this mobility matrix of second-rank tensors is also symmetric, i.e.

$$
a_{i j}^{\alpha \beta}=a_{j i}^{\beta \alpha}, \quad \tilde{b}_{i j}^{\alpha \beta}=b_{j i}^{\beta \alpha}, \quad c_{i j}^{\alpha \beta}=c_{j i}^{\beta \alpha} .
$$

As in the resistance problem, the two-sphere symmetry allows the following decompositions of the mobility tensors into scalar functions:

$$
\begin{aligned}
& \mathbf{a}^{\alpha \beta}=x_{\alpha \beta}^{a} \mathbf{e e}+y_{\alpha \beta}^{a}(\mathbf{I}-\mathbf{e e}) \\
& \mathbf{b}^{\alpha \beta}=-\tilde{\mathbf{b}}^{\beta \alpha}=y_{\alpha \beta}^{b} \mathbf{e} \times \mathbf{I} \\
& \mathbf{c}^{\alpha \beta}=x_{\alpha \beta}^{c} \mathbf{e}+y_{\alpha \beta}^{c}(\mathbf{I}-\mathbf{e e}) .
\end{aligned}
$$

Note that the scalar mobility functions are denoted by lower-case letters and superscripts, in contrast to the upper-case letters and superscripts used for the resistance functions.

The non-dimensionalizations of the mobility tensors are as follows, again using the caret notation:

$$
\begin{aligned}
& \hat{\mathbf{a}}^{\alpha \beta}=3 \pi\left(a_{\alpha}+a_{\beta}\right) \mathbf{a}^{\alpha \beta} \\
& \hat{\mathbf{b}}^{\alpha \beta}=\pi\left(a_{\alpha}+a_{\beta}\right)^{2} \mathbf{b}^{\alpha \beta} \\
& \hat{\mathbf{c}}^{\alpha \beta}=\pi\left(a_{\alpha}+a_{\beta}\right)^{3} \mathbf{c}^{\alpha \beta} .
\end{aligned}
$$

Similar to the resistance problem, these non-dimensional mobility tensors and their scalar functions depend on $s$ and $\lambda$ as defined in eq. (8). Also, relations between the non-dimensional scalar mobility functions that are analogous to eq. (10) can be written down, showing that there will be 10 independent mobility functions to be determined for $2 \leqslant s<\infty$ and $0 \leqslant \lambda<\infty$.

Note that the resistance matrix defined by eq. (4) and the mobility matrix defined by eq. (11) are reciprocal (inverse) of each other. The detailed transformation relations between the non-dimensional scalar resistance and mobility functions can be found in Jeffrey and Onishi (1984).

\section{METHOD OF TWIN MULTIPOLE EXPANSIONS}

We consider the slow motion of two spherical particles in an unbounded Newtonian and incompressible fluid. The particles are allowed to differ in radius 
and the fluid may slip at the surfaces of the particles. For the quasi-steady-state case, the velocity field $\mathbf{v}$ and dynamic pressure field $p$ satisfy the Stokes equations,

$$
\eta \nabla^{2} \mathbf{v}-\nabla p=\mathbf{0}, \quad \nabla \cdot \mathbf{v}=0 .
$$

The boundary conditions require that there be no relative normal flow at the surface of each sphere and that the tangential velocity of the fluid relative to the sphere at a point on its surface be proportional to the tangential stress prevailing at that point. If two sets of spherical coordinates $\left(r_{\alpha}, \theta_{\alpha}, \phi\right)$ are employed $(\alpha=1,2)$ to describe the two-sphere geometry following Happel and Brenner (1983) and Jeffrey and Onishi (1984), the boundary conditions at the particle surfaces for the general case are

$$
\begin{aligned}
r_{\alpha}=a_{\alpha}: \quad \mathbf{u}_{\alpha}\left(\theta_{\alpha}, \phi\right) & \equiv \mathbf{v}-\frac{1}{\beta_{\alpha}}\left(\mathbf{I}-\mathbf{e}_{\alpha} \mathbf{e}_{\alpha}\right) \mathbf{e}_{\alpha}: \tau \\
& =\mathbf{U}_{\alpha}+a_{x} \mathbf{\Omega}_{\alpha} \times \mathbf{e}_{\alpha}
\end{aligned}
$$

where $\tau$ is the fluid stress tensor, $\mathbf{e}_{\alpha}$ is the unit vector in the direction of $r_{\alpha}$, and $1 / \beta_{\alpha}$ is the slip coefficient about the surface of sphere $\alpha$.

The method of twin multipole expansions will be used to solve eqs (15) and (16). The pressure and velocity fields can be written as the sum of the contributions of singularities at the centers of the particles using Lamb's (1945) general solution:

$$
p=p^{(1)}+p^{(2)} \text { and } \mathbf{v}=\mathbf{v}^{(1)}+\mathbf{v}^{(2)}
$$

where

$$
\begin{aligned}
p^{(\alpha)}= & \eta \sum_{m=0}^{\infty} \sum_{n=m}^{x_{1}} \frac{1}{a_{\alpha}} p_{m n}^{(\alpha)}\left(\frac{a_{\alpha}}{r_{\alpha}}\right)^{n+1} Y_{m n}\left(\theta_{\alpha}, \phi\right) \\
\mathbf{v}^{(\alpha)}= & \sum_{m=0}^{\alpha} \sum_{n=m}^{\infty}\left\{\nabla \times\left[\mathbf{r}_{\alpha} q_{m n}^{(\alpha)}\left(\frac{a_{\alpha}}{r_{\alpha}}\right)^{n+1} Y_{m n}\left(\theta_{\alpha}, \phi\right)\right]\right. \\
& +a_{\alpha} \nabla\left[v_{m n}^{(\alpha)}\left(\frac{a_{\alpha}}{r_{\alpha}}\right)^{n+1} Y_{m n}\left(\theta_{\alpha}, \phi\right)\right] \\
& -\frac{n-2}{2 n(2 n-1) a_{\alpha}} r_{\alpha}^{2} \nabla\left[p_{m n}^{(x)}\left(\frac{a_{\alpha}}{r_{\alpha}}\right)^{n+1}\right. \\
& \left.\times Y_{m n}\left(\theta_{\alpha}, \phi\right)\right]+\frac{n+1}{n(2 n-1) a_{\alpha}} \\
& \left.\times \mathbf{r}_{\alpha} p_{m n}^{(\alpha)}\left(\frac{a_{\alpha}}{r_{\alpha}}\right)^{n+1} Y_{m n}\left(\theta_{\alpha}, \phi\right)\right\}
\end{aligned}
$$

and $Y_{m n}\left(\theta_{\alpha}, \phi\right)=P_{n}^{m}\left(\cos \theta_{\alpha}\right) \exp (\operatorname{im} \phi)$ are spherical surface harmonics. The coefficients $p_{m n}^{(\alpha)}, q_{m n}^{(\alpha)}$ and $v_{m n}^{(\alpha)}$ are functions only of $s$ and $\lambda$ and are to be determined from the boundary conditions.

To simplify the application of the boundary conditions, we follow Happel and Brenner (1983) and Jeffrey and Onishi (1984) in first constructing the following three scalar equations:

$$
\mathbf{e}_{\alpha} \cdot \mathbf{u}_{\alpha}=\sum_{m=0}^{\infty} \sum_{n=m}^{\infty} \chi_{m n}^{(\alpha)} Y_{m n}\left(\theta_{\alpha}, \phi\right)
$$

$$
\begin{array}{r}
-a_{\alpha} \nabla \cdot \mathbf{u}_{\alpha}=\sum_{m=0}^{\infty} \sum_{n=m}^{\infty} \psi_{m n}^{(\alpha)} Y_{m n}\left(\theta_{\alpha}, \phi\right) \\
a_{\alpha} \mathbf{e}_{\alpha} \cdot\left(\nabla \times \mathbf{u}_{\alpha}\right)=\sum_{m=0}^{\infty} \sum_{n=m}^{\infty} \omega_{m n}^{(\alpha)} Y_{m n}\left(\theta_{\alpha}, \phi\right)
\end{array}
$$

where the coefficients $\chi_{m n}^{(\alpha)}, \psi_{m n}^{(\alpha)}$ and $\omega_{m n}^{(\alpha)}$ can be evaluated from the boundary conditions given by eq. (16). Substituting eqs (16)-(18) into eq. (19), using the transformation rules for the two spherical coordinate systems, and equating coefficients of $Y_{m n}\left(\theta_{\alpha}, \phi\right)$, one obtains the expressions for functions $p_{m n}^{(\alpha)}, q_{m n}^{(\alpha)}$ and $v_{m n}^{(\alpha)}$ in terms of $\chi_{m n}^{(\alpha)}, \psi_{m n}^{(\alpha)}$ and $\omega_{m n}^{(\alpha)}$. The result includes three recurrence relations:

$$
\begin{aligned}
(n+ & +1)(2 n+1)\left[1+(2 n+1) \hat{\beta}_{\alpha}^{-1}\right] v_{m n}^{(\alpha)} \\
& -\frac{1}{2}(n+1)\left[1+(2 n+1) \hat{\beta}_{\alpha}^{-1}\right] p_{m n}^{(\alpha)} \\
& +\sum_{s=m}^{\alpha}\left(\begin{array}{c}
n+s \\
n+m
\end{array}\right) t_{\alpha}^{n-1} t_{3-\alpha}^{s}\left\{-(-1)^{\alpha} m\left(4 n^{2}-1\right)\right. \\
& \times \hat{\beta}_{3-\alpha}^{-1} \mathrm{i} q_{m s}^{(3-\alpha)} t_{3-\alpha}-n\left(4 n^{2}-1\right) \hat{\beta}_{3-\alpha}^{-1} v_{m s}^{(3-\alpha)} t_{3-\alpha}^{2} \\
& +\frac{n}{2 n+3}\left[1-\frac{1}{2}(2 n+1)^{2} \hat{\beta}_{3-\alpha}^{-1}\right] p_{m s}^{(3-\alpha)} t_{\alpha}^{2} \\
& -(2 n+1) \hat{\beta}_{3-\alpha}^{-1} \\
& \times \frac{2 m^{2}(-n s+2 n+2 s-1)-n s(2 n s-n-s+2)}{2 s(2 s-1)(n+s)} \\
& \left.\times p_{m s}^{(3-\alpha)}\right\} \\
& =\psi_{m n}^{(\alpha)}-\left[n-1+\left(2 n^{2}+1\right) \hat{\beta}_{\alpha}^{-1}\right] \chi_{m n}^{(\alpha)}
\end{aligned}
$$$$
\frac{n+1}{2 n-1}\left[1+(2 n+1) \hat{\beta}_{\alpha}^{-1}\right] p_{m n}^{(\alpha)}
$$$$
+\sum_{s=m}^{\infty}\left(\begin{array}{c}
n+s \\
n+m
\end{array}\right) t_{\alpha}^{n-1} t_{3-\alpha}^{s}
$$$$
\times\left\{(-1)^{\alpha} m(2 n+1)\left(1+2 \hat{\beta}_{3-\alpha}^{-1}\right)\right.
$$$$
\times \mathrm{i} q_{m s}^{(3-x)} t_{3-x}+n(2 n+1)\left(1+2 \hat{\beta}_{3-x}^{-1}\right)
$$$$
\times v_{m s}^{(3-\alpha)} t_{3-\alpha}^{2}+\frac{1}{2} n p_{m s}^{(3-\alpha)} t_{\alpha}^{2}+\frac{2 n+1}{2 n-1}\left(1+2 \hat{\beta}_{3-\alpha}^{-1}\right)
$$$$
\times \frac{2 m^{2}(-n s+2 n+2 s-1)-n s(2 n s-n-s+2)}{2 s(2 s-1)(n+s)}
$$$$
\left.\times p_{m s}^{(3-x)}\right\}
$$$$
=\psi_{m n}^{(\alpha)}+(n+2)\left(1+2 n \hat{\beta}_{\alpha}^{-1}\right) \chi_{m n}^{(\alpha)},
$$

$n(n+1)\left[1+(n+2) \hat{\beta}_{\alpha}^{-1}\right] q_{m n}^{(\alpha)}$

$$
\begin{aligned}
& +\left[1-(n-1) \hat{\beta}_{3-\alpha}^{-1}\right] \sum_{s=m}^{\infty}\left(\begin{array}{c}
n+s \\
n+m
\end{array}\right) t_{\alpha}^{n} t_{3-\alpha}^{s} \\
& \times\left\{-n s q_{m s}^{(3-\alpha)} t_{3-\alpha}+(-1)^{\alpha} \frac{m}{s} \mathrm{i} p_{m s}^{(3-\alpha)}\right\}=\omega_{m n}^{(\alpha)},
\end{aligned}
$$


where $t_{\alpha}=a_{\alpha} / r, \quad \mathrm{i}=\sqrt{-1}$ and $\hat{\beta}_{\alpha}=\beta_{\alpha} a_{\alpha} / \eta$. In the following analysis, $\hat{\beta}_{1}=\hat{\beta}_{2}=\hat{\beta}$ will be assumed.

The drag force and torque exerted by the fluid on each sphere can be expressed in a Cartesian coordinate system with unit vectors $(\mathbf{i}, \mathbf{j}, \mathbf{e})$, where the $\mathbf{i}$-axis is chosen in the plane $\phi=0$. Then

$$
\begin{aligned}
& \mathbf{F}_{\alpha}=-4 \pi \eta a_{\alpha}\left[p_{01}^{(\alpha)}(-1)^{3-\alpha} \mathbf{e}-p_{11}^{(\alpha)}(\mathbf{i}+\mathbf{j})\right] \\
& \mathbf{T}_{\alpha}=-8 \pi \eta a_{\alpha}^{2}\left[q_{01}^{(\alpha)}(-1)^{3-\alpha} \mathbf{e}-q_{11}^{(\alpha)}(\mathbf{i}+\mathbf{j})\right] .
\end{aligned}
$$

In the following two sections, eqs (19)-(21) will be applied to solve the 10 non-dimensional scalar resistance functions and the 10 mobility functions. Our solutions contain as special cases the solutions for two no-slip spheres $(\hat{\beta} \rightarrow \infty)$ presented in Jeffrey and Onishi (1984) and the solutions for two spherical gas bubbles $(\hat{\beta}=0)$.

\section{THE NON-DIMENSIONAL RESISTANCE FUNCTIONS}

\section{1. $\hat{\mathrm{X}}_{\alpha \beta}^{\mathrm{A}}(\mathrm{s}, \lambda)$}

To determine the non-dimensional resistance function $\hat{X}_{\alpha \beta}^{A}(s, \lambda)$, it is convenient to consider two particular problems. In the first problem, the velocities $U_{1}$ and $U_{2}$ of the two spheres are along the line through their centers with equal magnitudes but opposite directions, i.e.

$$
\mathbf{U}_{1}=-\mathbf{U}_{2}=U \mathbf{e}
$$

For the second problem, the spheres move along the line of centers with identical velocities,

$$
\mathbf{U}_{1}=\mathbf{U}_{2}=U \mathbf{e} .
$$

In either case, there is no particle rotation $\left(\boldsymbol{\Omega}_{1}=\boldsymbol{\Omega}_{2}=\mathbf{0}\right)$.

For the first problem, the coefficients defined in eq. (19) become

$$
\chi_{m n}^{(\alpha)}=U \delta_{m 0} \delta_{n 1}, \quad \psi_{m n}^{(\alpha)}=0, \quad \omega_{m n}^{(\alpha)}=0
$$

Note that these coefficients are the same for each sphere. In eq. (20), only the functions for $m=0$ will be non-zero due to the axial symmetry. Also, since the angular velocities are non-existent, all the functions $q_{m n}^{(\alpha)}$ are zero. Utilizing eqs (20) and (24), the functions $p_{0 n}^{(\alpha)}$ and $v_{0 n}^{(\alpha)}$ can be expanded as double power series in $t_{\alpha}=a_{\alpha} / r$ :

$$
\begin{gathered}
p_{0 n}^{(\alpha)}=\frac{3}{2} U \sum_{p=0}^{\infty} \sum_{q=0}^{\infty} P_{n p q} t_{\alpha}^{p} t_{3-\alpha}^{q} \\
v_{0 n}^{(\alpha)}=\frac{3}{4} U \sum_{p=0}^{\infty} \sum_{q=0}^{\infty} \frac{1}{2 n+1} V_{n p q} t_{\alpha}^{p} t_{3-\alpha}^{q} .
\end{gathered}
$$

The recurrence relations for the coefficients $P_{n p q}$ and $V_{n p q}$ are

$$
P_{n 00}=\delta_{n 1} \beta_{23}, \quad V_{n 00}=\delta_{n 1} \beta_{03}
$$

and

$$
\begin{aligned}
P_{n p q}= & \frac{n(2 n-1)}{2(n+1)\left[1+(2 n+1) \hat{\beta}^{-1}\right]} \sum_{s=1}^{q}\left(\begin{array}{c}
n+s \\
n
\end{array}\right) \\
& \times\left\{\beta_{20} \frac{(2 n+1)(2 n s-n-s+2)}{(2 n-1)(2 s-1)(n+s)}\right. \\
& \times P_{s(q-s)(p-n+1)-P_{s(q-s)(p-n-1)}} \\
& \left.-\beta_{20} \frac{2 n+1}{2 s+1} V_{s(q-s-2)(p-n+1)}\right\} \\
V_{n p q}= & P_{n p q}-\frac{n}{(n+1)\left[1+(2 n+1) \hat{\beta}^{-1}\right]} \sum_{s=1}^{q}\left(\begin{array}{c}
n+s \\
n
\end{array}\right) \\
& \times\left\{(2 n+1) \hat{\beta}^{-1} \frac{2 n s-n-s+2}{(2 s-1)(n+s)}\right. \\
& \times P_{s(q-s)(p-n+1)} \\
& +\frac{2-(2 n+1)^{2} \hat{\beta}^{-1}}{2 n+3} P_{s(q-s)(p-n-1)} \\
& \left.-\frac{\left(4 n^{2}-1\right) \hat{\beta}^{-1}}{2 s+1} V_{s(q-s-2)(p-n+1)}\right\}
\end{aligned}
$$

where $\beta_{n m}=(\hat{\beta}+n) /(\hat{\beta}+m) ; P_{n p q}=V_{n p q}=0$ if $p$ or $q$ is negative. After the substitution of eq. (25a), the force equation (21a) yields

$$
\hat{X}_{11}^{A}-\frac{1}{2}(1+\lambda) \hat{X}_{12}^{A}=\sum_{p=0}^{\infty} \sum_{q=0}^{\infty} P_{1 p q} t_{1}^{p} t_{2}^{q} .
$$

For the second problem, the quantities defined in eq. (19) become

$$
\chi_{m n}^{(\alpha)}=(-1)^{3-\alpha} U \delta_{m 0} \delta_{n 1}, \quad \psi_{m n}^{(\alpha)}=0, \quad \omega_{m n}^{(\alpha)}=0 .
$$

By substituting these conditions into eq. (20), it is found that the functions $p_{0 n}^{(\alpha)}$ and $v_{0 n}^{(\alpha)}$ can be expanded in the form of eq. (25) with coefficients $P_{n p q}$ and $V_{n p q}$ being replaced by $(-1)^{n+p+q+\alpha} P_{n p q}$ and $(-1)^{n+p+q+\alpha} V_{n p q}$, respectively. Thus, it is easy to conclude that

$$
\hat{X}_{11}^{A}+\frac{1}{2}(1+\lambda) \hat{X}_{12}^{A}=\sum_{p=0}^{\infty} \sum_{q=0}^{\infty}(-1)^{p+q} P_{1 p q} t_{1}^{p} t_{2}^{q} .
$$

From eqs (28) and (30) it is obvious that $\hat{X}_{11}^{A}$ is a series only of terms in which $p+q$ is even and $\hat{X}_{12}^{A}$ is a series only of terms in which $p+q$ is odd. Their results as functions of $s$ and $\lambda$ have the same form as that given in Jeffrey and Onishi (1984),

$$
\begin{aligned}
& \hat{X}_{11}^{A}=\sum_{k=0}^{\infty} f_{2 k}(\lambda)(1+\lambda)^{-2 k} s^{-2 k} \\
& \hat{X}_{12}^{A}=-2 \sum_{k=0}^{\infty} f_{2 k+1}(\lambda)(1+\lambda)^{-2 k-2} s^{-2 k-1}
\end{aligned}
$$

where

$$
f_{k}(\lambda)=2^{k} \sum_{q=0}^{k} P_{1(k-q) q} \lambda^{q}
$$


Explicitly,

$$
\begin{aligned}
& f_{0}=\beta_{23}, \quad f_{1}=3 \beta_{23}^{2} \lambda, \quad f_{2}=9 \beta_{23}^{3} \lambda \\
& f_{3}=-4 \beta_{03} \beta_{23} \lambda+27 \beta_{23}^{4} \lambda^{2}-4 \beta_{03} \beta_{23} \lambda^{3} \\
& f_{4}=-24 \beta_{03} \beta_{23}^{2} \lambda+81 \beta_{23}^{5} \lambda^{2} \\
& +36\left(1+5 \hat{\beta}^{-1}+10 \hat{\beta}^{-2}\right) \beta_{03} \beta_{05} \beta_{23}^{2} \lambda^{3} \\
& f_{5}=72\left(1+5 \hat{\beta}^{-1}+15 \hat{\beta}^{-2}\right) \beta_{03} \beta_{05} \beta_{23}^{3} \lambda^{2}+243 \beta_{23}^{6} \lambda^{3} \\
& +72\left(1+5 \hat{\beta}^{-1}+15 \hat{\beta}^{-2}\right) \beta_{03} \beta_{05} \beta_{23}^{3} \lambda^{4} \\
& f_{6}=16 \beta_{03}^{2} \beta_{23} \hat{\lambda}+108\left(1+5 \hat{\beta}^{-1}+30 \hat{\beta}^{-2}\right) \beta_{03} \beta_{05} \beta_{23}^{4} \hat{\lambda}^{2} \\
& +\left(729 \beta_{23}^{7}+32 \beta_{03}^{2} \beta_{23}-480 \beta_{05} \beta_{23}^{2}\right) \lambda^{3} \\
& +648\left(1+5 \hat{\beta}^{-1}+10 \hat{\beta}^{-2}\right) \beta_{03} \beta_{05} \beta_{23}^{4} \hat{\lambda}^{4} \\
& +\frac{144}{5} \beta_{23}^{2}\left(\beta_{(-2) 3}-10 \beta_{05}+14 \beta_{27}\right) \lambda^{5} \\
& f_{7}=\frac{144}{5} \beta_{23}^{3}\left[3 \beta_{(-2) 3}+42 \beta_{27}\right. \\
& \left.-5\left(7+35 \hat{\beta}^{-1}+46 \hat{\beta}^{-2}\right) \beta_{02} \beta_{03} \beta_{05}\right] \lambda^{2} \\
& +1620\left(1+5 \hat{\beta}^{-1}+12 \hat{\beta}^{-2}\right) \beta_{03} \beta_{05} \beta_{23}^{5} \lambda^{3} \\
& +3 \beta_{23}^{2}\left[729 \beta_{23}^{6}+64 \beta_{03}^{2}-720 \beta_{05} \beta_{23}\right. \\
& \left.+48\left(1+5 \hat{\beta}^{-1}+10 \hat{\beta}^{-2}\right)\left(10 \beta_{05} \beta_{23}-\beta_{03}^{2}\right)\right] \hat{\lambda}^{4} \\
& +1620\left(1+5 \hat{\beta}^{-1}+12 \widehat{\beta}^{-2}\right) \beta_{03} \beta_{05} \beta_{23}^{5} \lambda^{5} \\
& +\frac{144}{5} \beta_{23}^{3}\left[3 \beta_{(-2) 3}+42 \beta_{27}-5\left(7+35 \hat{\beta}^{-1}\right.\right. \\
& \left.\left.+46 \hat{\beta}^{-2}\right) \beta_{02} \beta_{03} \beta_{05}\right] \lambda^{6} \text {. }
\end{aligned}
$$

In the limit of perfect slip at the particle surfaces ( $\hat{\beta}=0$ or spherical gas bubbles),

$$
\begin{aligned}
f_{8}= & \frac{512}{3}\left(\lambda^{2}+2 \lambda^{3}+5 \lambda^{4}+3 \lambda^{5}+3 \lambda^{6}+\lambda^{7}\right) \\
f_{9}= & \frac{1024}{3}\left(\lambda^{2}+3 \lambda^{3}+6 \lambda^{4}+8 \lambda^{5}+6 \lambda^{6}+3 \lambda^{7}+\lambda^{8}\right) \\
f_{10}= & \frac{2048}{3}\left(\lambda^{2}+3 \lambda^{3}+9 \lambda^{4}+11 \lambda^{5}+14 \lambda^{6}+6 \lambda^{7}\right. \\
& \left.+4 \lambda^{8}+\lambda^{9}\right) \\
f_{11}= & \frac{4096}{3}\left(\lambda^{2}+4 \lambda^{3}+10 \lambda^{4}+20 \lambda^{5}+21 \lambda^{6}+20 \lambda^{7}\right. \\
& \left.+10 \lambda^{8}+4 \lambda^{9}+\lambda^{10}\right) .
\end{aligned}
$$

The formulas of $f_{8}, f_{9}, f_{10}$ and $f_{11}$ for the special case of no-slip spheres $(\hat{\beta} \rightarrow \infty)$ were given by Jeffrey and Onishi (1984). Expressions for $f_{k}(\lambda)$ with higher orders can be explicitly derived if desired.

Using a method of reflections, Hetsroni and Haber (1978) derived the explicit formulas for the resistance functions $\hat{X}_{11}^{A}, \hat{X}_{12}^{A}, \hat{Y}_{11}^{A}$ and $\hat{X}_{12}^{A}$ in power series of $1 / r$ up to $O\left(r^{-5}\right)$ for the case of two fluid drops with arbitrary radii. The above expressions agree with (and are much more accurate than) their results (taking the viscosities of the drops or $\eta^{*}$ as zero) in the limit $\hat{\beta}=0$. It can also be found that the interaction between two slip spheres with a finite value of $\hat{\beta}$ is different from that between two fluid drops with

\begin{tabular}{|c|c|c|c|c|}
\hline \multirow{2}{*}{$\frac{\beta a}{\eta}$} & \multirow{2}{*}{$\frac{2 a}{r}$} & \multicolumn{2}{|c|}{ Asymptotic solution } & \multirow{2}{*}{$\begin{array}{l}\text { Exact } \\
\text { solution }\end{array}$} \\
\hline & & $O\left(s^{-7}\right)$ & $O\left(s^{-11}\right)$ & \\
\hline \multirow[t]{9}{*}{0} & 0.2 & 0.9092 & 0.9092 & 0.9092 \\
\hline & 0.4 & 0.8343 & 0.8345 & 0.8345 \\
\hline & 0.6 & 0.7714 & 0.7741 & 0.7742 \\
\hline & 0.8 & 0.7099 & 0.7260 & 0.7276 \\
\hline & 0.9 & 0.6710 & 0.7027 & 0.7090 \\
\hline & 0.95 & 0.6466 & 0.6888 & 0.7008 \\
\hline & 0.99 & 0.6235 & 0.6753 & 0.6946 \\
\hline & 0.999 & 0.6178 & 0.6718 & 0.6933 \\
\hline & 0.9999 & 0.6173 & 0.6714 & 0.6931 \\
\hline \multirow[t]{9}{*}{1} & 0.2 & 0.8992 & & 0.8992 \\
\hline & 0.4 & 0.8189 & & 0.8191 \\
\hline & 0.6 & 0.7538 & & 0.7575 \\
\hline & 0.8 & 0.6887 & & 0.7122 \\
\hline & 0.9 & 0.6438 & & 0.6947 \\
\hline & 0.95 & 0.6142 & & 0.6869 \\
\hline & 0.99 & 0.5856 & & 0.6811 \\
\hline & 0.999 & 0.5784 & & 0.6798 \\
\hline & 0.9999 & 0.5776 & & 0.6797 \\
\hline \multirow[t]{9}{*}{10} & 0.2 & 0.8792 & & 0.8792 \\
\hline & 0.4 & 0.7889 & & 0.7894 \\
\hline & 0.6 & 0.7184 & & 0.7262 \\
\hline & 0.8 & 0.6323 & & 0.6838 \\
\hline & 0.9 & 0.5552 & & 0.6680 \\
\hline & 0.95 & 0.4988 & & 0.6610 \\
\hline & 0.99 & 0.4416 & & 0.6559 \\
\hline & 0.999 & 0.4270 & & 0.6548 \\
\hline & 0.9999 & 0.4255 & & 0.6547 \\
\hline \multirow[t]{9}{*}{$\infty$} & 0.2 & 0.8706 & 0.8706 & 0.8706 \\
\hline & 0.4 & 0.7763 & 0.7772 & 0.7772 \\
\hline & 0.6 & 0.7016 & 0.7136 & 0.7140 \\
\hline & 0.8 & 0.5906 & 0.6618 & 0.6729 \\
\hline & 0.9 & 0.4763 & 0.6151 & 0.6578 \\
\hline & 0.95 & 0.3895 & 0.5723 & 0.6512 \\
\hline & 0.99 & 0.3001 & 0.5206 & 0.6463 \\
\hline & 0.999 & 0.2771 & 0.5060 & 0.6453 \\
\hline & 0.9999 & 0.2747 & 0.5045 & 0.6452 \\
\hline
\end{tabular}
a value of $3 \eta^{*}$ equal to $\hat{\beta}$, although the flow field induced by an isolated slip sphere is equivalent to the external flow field caused by an isolated fluid drop under this condition.
The exact (numerical) solution of the resistance functions $\hat{X}_{11}^{A}$ and $\hat{X}_{12}^{A}$ was obtained by solving the problem of axisymmetric translational motion of two slip spheres using bipolar coordinates (Chen and Keh, 1995). Table 1 gives a comparison of our asymptotic solution from the method of twin multipole expansions with this exact solution. For simplicity, only the case of two identical spheres $\left(a_{1}=a_{2}=a\right.$ and $\left.\beta_{1}=\beta_{2}=\beta\right)$ with equal velocities $\left(\mathbf{U}_{1}=\mathbf{U}_{2}=U \mathbf{e}\right)$ is presented. In this specific case, the particles will experience the same drag force $\left(F_{1}=F_{2}=F\right)$ because $\hat{X}_{11}^{A}=\hat{X}_{22}^{A}$ and $\hat{X}_{12}^{A}=\hat{X}_{21}^{A}$. It is found from Table 1 that the predictions of $F / F^{(0)}\left[=\left(\hat{X}_{11}^{A}+\hat{X}_{12}^{A}\right)\right.$ $(\beta a+2 \eta) /(\beta a+3 \eta)]$ from the asymptotic solution for various values of $\beta a / \eta$ are in good agreement with those of the exact solution. The errors in drag forces

Table 1. The normalized drag forces $F / F^{(0)}$ experienced by two identical spheres translating with equal velocities along the line of their centers 
are less than $1.8 \%$ for cases $2 a / r \leqslant 0.6$ if the asymptotic formula (31) is accurate to $O\left(s^{-7}\right)$ or less than $6.5 \%$ for cases $2 a / r \leqslant 0.9$ if eq. (31) is accurate to $O\left(s^{-11}\right)$. For the situation of two slip spheres with different radii, eq. (31) can also be found to agree well with the exact solution. In general, the accuracy of eq. (31) with finite terms decreases monotonically with the increase of $\beta a / \eta$ for a given value of $s$. In Section 5.1, one will see that the asymptotic results for the corresponding mobility problem of the same order of $s^{-1}$ obtained from the same method are much more accurate than the resistance results presented here.

\section{2. $\hat{\mathrm{Y}}_{\alpha \beta}^{\mathrm{A}}(\mathrm{s}, \lambda)$}

We now consider two problems of the motion of two spheres normal to the line of their centers. In the first problem,

$$
\mathbf{U}_{1}=-\mathbf{U}_{2}=U \mathbf{i}
$$

and in the second problem,

$$
\mathbf{U}_{1}=\mathbf{U}_{2}=U \mathbf{i}
$$

with in each case $\boldsymbol{\Omega}_{1}=\boldsymbol{\Omega}_{2}=\mathbf{0}$.

For the first problem, one has the coefficients in eq. (19) as

$$
\chi_{m n}^{(\alpha)}=(-1)^{\alpha} U \delta_{m 1} \delta_{n 1}, \quad \psi_{m n}^{(\alpha)}=0, \quad \omega_{m n}^{(\alpha)}=0 .
$$

In eq. (20), only the functions for $m=1$ will be nonzero now. The expansions being used this time are

$$
\begin{aligned}
& p_{1 n}^{(\alpha)}=(-1)^{\alpha} \frac{3}{2} U \sum_{p=0}^{\infty} \sum_{q=0}^{\infty} P_{n p q} t_{\alpha}^{p} t_{3-\alpha}^{q} \\
& q_{1 n}^{(\alpha)}=-\mathrm{i} U \sum_{p=0}^{\infty} \sum_{q=0}^{\infty} Q_{n p q} t_{\alpha}^{p} t_{3-\alpha}^{q} \\
& v_{1 n}^{(\alpha)}=(-1)^{\alpha} \frac{3}{4} U \sum_{p=0}^{\infty} \sum_{q=0}^{\infty} \frac{1}{2 n+1} V_{n p q} t_{\alpha}^{p} t_{3-\alpha}^{q} .
\end{aligned}
$$

The recurrence relations for coefficients $P_{n p q}, Q_{n p q}$ and $V_{n p q}$, are

$$
P_{n 00}=\delta_{n 1} \beta_{23}, \quad Q_{n 00}=0, \quad V_{n 00}=\delta_{n 1} \beta_{03}
$$

and

$$
\begin{aligned}
P_{n p q}= & \frac{2 n-1}{(n+1)\left[1+(2 n+1) \hat{\beta}^{-1}\right]} \sum_{s=1}^{q}\left(\begin{array}{c}
n+s \\
n+1
\end{array}\right) \\
& \times\left\{\beta_{20} \frac{2 n+1}{2 n-1} \frac{(n+s)(n s+4)-2(n s+1)^{2}}{2 s(2 s-1)(n+s)}\right. \\
& \times P_{s(q-s)(p-n+1)}+\frac{n}{2} P_{s(q-s)(p-n-1)} \\
& +\beta_{20} \frac{n(2 n+1)}{2(2 s+1)} V_{s(q-s-2)(p-n+1)} \\
& \left.-\frac{2}{3} \beta_{20}(2 n+1) Q_{s(q-s-1)(p-n+1)}\right\}
\end{aligned}
$$

$$
\begin{aligned}
Q_{n p q}= & \frac{1-(n-1) \hat{\beta}^{-1}}{(n+1)\left[1+(n+2) \hat{\beta}^{-1}\right]} \sum_{s=1}^{q}\left(\begin{array}{c}
n+s \\
n+1
\end{array}\right) \\
& \times\left\{-\frac{3}{2 n s} P_{s(q-s)(p-n)}+s Q_{s(q-s-1)(p-n)}\right\}
\end{aligned}
$$

$$
\begin{aligned}
V_{n p q}= & P_{n p q}-\frac{n}{(n+1)\left[1+(2 n+1) \hat{\beta}^{-1}\right]} \\
& \times \sum_{s=1}^{q}\left(\begin{array}{l}
n+s \\
n+1
\end{array}\right)\left\{\frac{(2 n+1) \hat{\beta}^{-1}}{n}\right. \\
& \times \frac{(n+s)(n s+4)-2(n s+1)^{2}}{s(2 s-1)(n+s)} P_{s(q-s)(p-n+1)} \\
& -\frac{2-(2 n+1)^{2} \hat{\beta}^{-1}}{2 n+3} P_{s(q-s)(p-n-1)} \\
& +\frac{\left(4 n^{2}-1\right) \hat{\beta}^{-1}}{2 s+1} V_{s(q-s-2)(p-n+1)} \\
& \left.-\frac{4\left(4 n^{2}-1\right) \hat{\beta}^{-1}}{n} Q_{s(q-s-1)(p-n+1)}\right\}
\end{aligned}
$$

The force equation (21a) now leads to

$$
\hat{Y}_{11}^{A}-\frac{1}{2}(1+\lambda) \hat{Y}_{12}^{A}=\sum_{p=0}^{\infty} \sum_{q=0}^{\infty} P_{1 p q} t_{1}^{p} t_{2}^{q} .
$$

By considering the second problem and comparing the result with eq. (39), it can be found that $\hat{Y}_{11}^{A}$ consists of the even powers of $s$ and $\hat{Y}_{12}^{A}$ of the odd powers of $s$. The explicit results can again be written as

$$
\begin{aligned}
& \hat{Y}_{11}^{A}=\sum_{k=0}^{\infty} f_{2 k}(\lambda)(1+\lambda)^{-2 k} s^{-2 k} \\
& \hat{Y}_{12}^{A}=-2 \sum_{k=0}^{\infty} f_{2 k+1}(\lambda)(1+\lambda)^{-2 k-2} s^{-2 k-1} .
\end{aligned}
$$

Here, $f_{k}(\lambda)$ is given by the form eq. (32), and the first few values are

$$
\begin{aligned}
f_{0}= & \beta_{23}, \quad f_{1}=\frac{3}{2} \beta_{23}^{2} \lambda, \quad f_{2}=\frac{9}{4} \beta_{23}^{3} \lambda \\
f_{3}= & 2 \beta_{03} \beta_{23} \lambda+\frac{27}{8} \beta_{23}^{4} \lambda^{2}+2 \beta_{03} \beta_{23} \lambda^{3} \\
f_{4}= & 6 \beta_{03} \beta_{23}^{2} \lambda+\frac{81}{16} \beta_{23}^{5} \lambda^{2}+18 \beta_{03} \beta_{23}^{2} \lambda^{3} \\
f_{5}= & \frac{63}{2} \beta_{03} \beta_{23}^{3} \lambda^{2}+\frac{243}{32} \beta_{23}^{6} \lambda^{3}+\frac{63}{2} \beta_{03} \beta_{23}^{3} \lambda^{4} \\
f_{6}= & 4 \beta_{03}^{2} \beta_{23} \lambda+54 \beta_{03} \beta_{23}^{4} \lambda^{2} \\
& +\beta_{23}\left(\frac{729}{64} \beta_{23}^{6}+8 \beta_{03}^{2}\right) \lambda^{3}+81 \beta_{03} \beta_{23}^{4} \lambda^{4} \\
& +\frac{12}{5} \beta_{23}^{2}\left(3 \beta_{(-2) 3}+20 \beta_{(-1) 4}+7 \beta_{27}\right) \lambda^{5} \\
f_{7}= & \frac{18}{5} \beta_{23}^{3}\left(3 \beta_{(-2) 3}+20 \beta_{(-1) 4}+7 \beta_{27}+10 \beta_{02} \beta_{03}\right) \lambda^{2} \\
& +\frac{1053}{8} \beta_{03} \beta_{23}^{5} \lambda^{3}+\beta_{23}^{2}\left(\frac{2187}{128} \beta_{23}^{6}+132 \beta_{03}^{2}\right) \lambda^{4} \\
& +\frac{1053}{8} \beta_{03} \beta_{23}^{5} \lambda^{5}+\frac{18}{5} \beta_{23}^{3} \\
& \times\left(3 \beta_{(-2) 3}+20 \beta_{(-1) 4}+7 \beta_{27}+10 \beta_{02} \beta_{03}\right) \lambda^{6} .
\end{aligned}
$$


If $\hat{\beta}=0$,

$$
\begin{aligned}
f_{8}= & \frac{2}{15}\left(-40 \lambda^{2}+5 \lambda^{4}-80 \lambda^{6}-256 \lambda^{7}\right) \\
f_{9}= & \frac{2}{15}\left(-256 \lambda^{2}-80 \lambda^{3}+5 \lambda^{5}-80 \lambda^{7}-256 \lambda^{8}\right) \\
f_{10}= & \frac{2}{15}\left(-256 \lambda^{2}-80 \lambda^{3}+5 \lambda^{5}-120 \lambda^{7}\right. \\
& \left.-512 \lambda^{8}-1280 \lambda^{9}\right) \\
f_{11}= & \frac{2}{15}\left(-1280 \lambda^{2}-512 \lambda^{3}-120 \lambda^{4}+1925 \lambda^{6}\right. \\
& \left.-120 \lambda^{8}-512 \lambda^{9}-1280 \lambda^{10}\right) .
\end{aligned}
$$

The above expressions are consistent with and much more accurate than the method-of-reflections results obtained by Hetsroni and Haber (1978) for the motion of two fluid drops (taking $\eta^{*}=0$ ) in the case $\hat{\beta}=0$. For finite values of $\hat{\beta}$ and $\eta^{*}$, the formulas of $\hat{X}_{11}^{A}, \hat{X}_{12}^{A}, \hat{Y}_{11}^{A}$ and $\hat{Y}_{12}^{A}$ for the motion of two slip spheres and for the motion of two spherical drops are identical up to $O\left(r^{-3}\right)$ if one puts $\hat{\beta}=3 \eta^{*}$; however, this is no longer true when terms of $O\left(r^{-4}\right)$ are retained.

It can be found from eq. (40) that the interaction effect between two spheres translating perpendicular to the line of their centers is more significant when the slip coefficients at the particle surfaces become smaller (or when the value of $\hat{\beta}$ becomes larger). This behavior is consistent with that observed for the axisymmetric translation of two slip spheres.

\section{3. $\hat{\mathrm{Y}}_{\alpha \beta}^{\mathrm{B}}(\mathrm{s}, \lambda)$}

The two problems specified in eqs (33) and (34) in the preceding subsection can be used to find functions $\hat{Y}_{z \beta}^{B}(s, \lambda)$. Substituting eq. (36b) into eq. (21b) and applying the definition of the resistance functions, one obtains

$$
\hat{Y}_{11}^{B}-\frac{1}{4}(1+\lambda)^{2} \hat{Y}_{12}^{B}=2 \sum_{p=0}^{\infty} \sum_{q=0}^{\infty} Q_{1 p q} t_{1}^{p} t_{2}^{q}
$$

When the second problem is considered, it can be found that $\hat{Y}_{11}^{B}$ consists of the odd powers of $s$ and $\hat{Y}_{12}^{B}$ the even powers. Thus,

$$
\begin{aligned}
& \hat{Y}_{11}^{B}=\sum_{k=0}^{\infty} f_{2 k+1}(\lambda)(1+\lambda)^{-2 k-1} s^{-2 k-1} \\
& \hat{Y}_{12}^{B}=-4 \sum_{k=0}^{\infty} f_{2 k}(\lambda)(1+\lambda)^{-2 k-2} s^{-2 k} .
\end{aligned}
$$

Here,

$$
f_{k}(\lambda)=-2^{k+1} \sum_{q=0}^{k} Q_{1(k-q) q^{\lambda^{q}}}
$$

and some explicit expressions are

$$
\begin{aligned}
& f_{0}=f_{1}=0, \quad f_{2}=-6 \beta_{03} \beta_{23} \lambda, \quad f_{3}=-9 \beta_{03} \beta_{23}^{2} \lambda \\
& f_{4}=-\frac{27}{2} \beta_{03} \beta_{23}^{3} \lambda^{2} \\
& f_{5}=-12 \beta_{03}^{2} \beta_{23} \lambda-\frac{81}{4} \beta_{03} \beta_{23}^{4} \lambda^{2}-36 \beta_{03}^{2} \beta_{23} \lambda^{3} \\
& f_{6}=-108 \beta_{03}^{2} \beta_{23}^{2} \lambda^{2}-\frac{243}{8} \beta_{03} \beta_{23}^{5} \lambda^{3}-72 \beta_{03}^{2} \beta_{23}^{2} \lambda^{4}
\end{aligned}
$$

$$
\begin{aligned}
f_{7}= & -189 \beta_{03}^{2} \beta_{23}^{3} \lambda^{2}-3 \beta_{03}\left({ }_{16}^{243} \beta_{23}^{6}+160 \beta_{03} \beta_{25}\right) \lambda^{3} \\
& -243 \beta_{03}^{2} \beta_{23}^{3} \lambda^{4}-48 \beta_{23}^{2}\left(6 \beta_{02} \beta_{05}\right. \\
& \left.+4 \beta_{(-1) 2} \beta_{04}-7 \beta_{07}\right) \lambda^{5} .
\end{aligned}
$$

It can be seen that $\hat{Y}_{\alpha \beta}^{B}=0$ for all values of $s$ in the limit $\hat{\beta}=0$. This is expected since the torques exerted on gas bubbles by the surrounding fluid are zero.

\section{4. $\hat{\mathrm{X}}_{\alpha \beta}^{\mathrm{C}}(\mathrm{s}, \lambda)$}

To determine the functions $\hat{X}_{\alpha \beta}^{c}(s, \lambda)$, we consider two problems in which the two spheres rotate about the line of their centers. It is convenient to express the rotation in terms of a surface speed $U$ :

$$
a_{1} \mathbf{\Omega}_{1}=\mp a_{2} \boldsymbol{\Omega}_{2}=U \mathbf{e} .
$$

In each case, the translational velocities are zero $\left(\mathbf{U}_{1}=\mathbf{U}_{2}=\mathbf{0}\right)$. When the minus sign is taken, the coefficients defined in eq. (19) become

$$
\chi_{m n}^{(x)}=0, \quad \psi_{m n}^{(x)}=0, \quad \omega_{m n}^{(x)}=2 U \delta_{m 0} \delta_{n 1} . \quad(45 \mathrm{a}-\mathrm{c})
$$

The only non-zero functions in eq. (20) are $q_{0 n}^{(\alpha)}$, which can be expanded as

$$
q_{0 n}^{(x)}=U \sum_{p=0}^{\infty} \sum_{q=0}^{\infty} Q_{n p q} t_{\alpha}^{p} t_{3-\alpha}^{q} .
$$

The recurrence relations for coefficients $Q_{n p q}$ are

$Q_{n 00}=\delta_{n 1} \beta_{03}$

$$
Q_{n p q}=\frac{1-(n-1) \hat{\beta}^{-1}}{(n+1)\left[1+(n+2) \hat{\beta}^{-1}\right]} \sum_{s=1}^{q}\left(\begin{array}{c}
n+s \\
n
\end{array}\right)
$$$$
\times s Q_{s(q-s-1)(p-n)} \text {. }
$$

After substituting eq. (46) into the torque equation (21b), one obtains

$$
\hat{X}_{11}^{c}-\frac{(1+\lambda)^{3}}{8 \lambda} \hat{X}_{12}^{c}=\sum_{p=0}^{\infty} \sum_{q=0}^{\infty} Q_{1 p q} t_{1}^{p} t_{2}^{q} .
$$

When the plus sign in eq. (44) is considered and its torque result is compared with the above equation, it is easy to find that

$$
\begin{aligned}
& \hat{X}_{11}^{c}=\sum_{k=0}^{\infty} f_{2 k}(\lambda)(1+\lambda)^{-2 k} s^{-2 k} \\
& \hat{X}_{12}^{c}=-8 \sum_{k=0}^{\infty} f_{2 k+1}(\lambda)(1+\lambda)^{-2 k-4} s^{-2 k-1}
\end{aligned}
$$

where

$f_{k}(\hat{\lambda})=2^{k} \sum_{q=0}^{k} Q_{1(k-q) q} \lambda^{q+j}, j= \begin{cases}0 & \text { if } k \text { is even } \\ 1 & \text { if } k \text { is odd. }\end{cases}$

Explicitly,

$$
\begin{array}{ll}
f_{0}=\beta_{03}, & f_{1}=f_{2}=0 \\
f_{3}=8 \beta_{03}^{2} \lambda^{3}, & f_{4}=f_{5}=0 \\
f_{6}=64 \beta_{03}^{3} \lambda^{3}, & f_{7}=0 .
\end{array}
$$

If $\hat{\beta}=0, \hat{X}_{\alpha \beta}^{c}=0$ as expected. 
4.5. $\hat{\mathrm{Y}}_{\alpha \beta}^{\mathrm{C}}(\mathrm{s}, \lambda)$

The functions $\hat{Y}_{\alpha \beta}^{c}(s, \lambda)$ are obtained from problems of two spheres defined by

$$
a_{1} \mathbf{\Omega}_{1}= \pm a_{2} \mathbf{\Omega}_{2}=U \mathbf{i}
$$

with $\mathbf{U}_{1}=\mathbf{U}_{2}=\mathbf{0}$. When the plus sign is considered, we have

$$
\chi_{m n}^{(\alpha)}=0, \quad \psi_{m n}^{(\alpha)}=0, \quad \omega_{m n}^{(\alpha)}=(-1)^{\alpha} 2 U \delta_{m 1} \delta_{n 1} .
$$

The expansions (36) and the recurrence relations (38) can be used again in this case, but the initial conditions (37) are replaced by

$$
P_{n 00}=0, \quad Q_{n 00}=\delta_{n 1} \beta_{03}, \quad V_{n 00}=0 .
$$

From the torque exerted on a sphere given by eq. (21b) one finds

$$
\hat{Y}_{11}^{C}+\frac{(1+\lambda)^{3}}{8 \lambda} \hat{Y}_{12}^{C_{2}}=\sum_{p=0}^{\infty} \sum_{q=0}^{\infty} Q_{1 p q} t_{1}^{p} t_{2}^{q} .
$$

In the standard notation,

$$
\begin{aligned}
& \hat{Y}_{11}^{C_{1}}=\sum_{k=0}^{\infty} f_{2 k}(\lambda)(1+\lambda)^{-2 k} s^{-2 k} \\
& \hat{Y}_{12}^{C}=8 \sum_{k=0}^{\infty} f_{2 k+1}(\lambda)(1+\lambda)^{-2 k-4} s^{-2 k-1} .
\end{aligned}
$$

Here, $f_{k}(\lambda)$ is given by the form of eq. (50), and the first few values are

$f_{0}=\beta_{03}, \quad f_{1}=f_{2}=0$

$f_{3}=4 \beta_{03}^{2} \lambda^{3}, \quad f_{4}=12 \beta_{03}^{2} \beta_{23} \lambda, \quad f_{5}=18 \beta_{03}^{2} \beta_{23}^{2} \lambda^{4}$

$f_{6}=27 \beta_{03}^{2} \beta_{23}^{3} \lambda^{2}+16 \beta_{03}^{2}\left(\beta_{03}+15 \beta_{25}\right) \lambda^{3}$

$f_{7}=72 \beta_{03}^{3} \beta_{23} \lambda^{4}+\frac{81}{2} \beta_{03}^{2} \beta_{23}^{4} \lambda^{5}+72 \beta_{03}^{3} \beta_{23} \lambda^{6}$.

Again, $\hat{Y}_{\alpha \beta}^{c}=0$ in the limit $\hat{\beta}=0$.

\section{THE NON-DIMENSIONAL MOBILITY FUNCTIONS}

\section{1. $\hat{\mathrm{x}}_{\alpha \beta}^{\mathrm{a}}(\mathrm{s}, \lambda)$}

We now turn to the mobility functions $\hat{x}_{\alpha \beta}^{a}(s, \lambda)$ and consider the external forces acting on the two spheres given by

$$
\left(6 \pi \eta a_{1}\right)^{-1} \mathbf{F}_{1}=-\left(6 \pi \eta a_{2}\right)^{-1} \mathbf{F}_{2}=U \mathbf{e}
$$

where $U$ is the magnitude of velocity that either sphere would have when the two spheres are far away from each other. In addition, there is no torque exerted on the spheres $\left(\mathbf{T}_{1}=\mathbf{T}_{2}=\mathbf{0}\right)$. We wish to calculate the motion of the spheres, which is given by $\mathbf{U}_{1}=U_{1} \mathbf{e}, \mathbf{U}_{2}=-U_{2} \mathbf{e}$ and $\boldsymbol{\Omega}_{1}=\mathbf{\Omega}_{2}=\mathbf{0}$. Similar to the first problem considered for the resistance functions $\hat{X}_{\alpha \beta}^{A}(s, \lambda)$, the quantities defined in eq. (19) for this problem are given by eq. (24) in which $U$ is replaced by $U_{\alpha}$. Also, in eq. (20), only the functions $p_{m n}^{(\alpha)}$ and $v_{m n}^{(\alpha)}$ with $m=0$ are non-zero. The functions $p_{O n}^{(\alpha)}$, $v_{0 n}^{(\alpha)}$ and the particle velocities $U_{\alpha}$ can be expanded as eq. (25) and

$$
U_{x}=U \sum_{p=0}^{\infty} \sum_{q=0}^{\infty} U_{p q} t_{\alpha}^{p} t_{3-\alpha}^{q} .
$$

From eqs (21a), (25a) and (56) it is easy to know that

$$
P_{1 p q}=\delta_{p 0} \delta_{q 0}
$$

The recurrence relations for coefficients $P_{n p q}$ and $V_{n p q}$ in eq. (27) remain valid. Taking $n=1$ and using eqs (24), (25) and (57), eq. (20b) gives

$$
\begin{aligned}
U_{p q}= & \sum_{s=1}^{q} \frac{s+1}{4}\left\{-\frac{3}{2 s-1} P_{s(q-s) p}+\beta_{02} P_{s(q-s)(p-2)}\right. \\
& \left.+\frac{3}{2 s+1} V_{s(q-s-2) p}\right\}
\end{aligned}
$$

which allows us to calculate the coefficients $U_{p q}$ from using eqs (58) and (27). Using eqs (21a), (25a), (57) and (58), the mobility functions are related to these coefficients by

$$
\hat{x}_{11}^{a}-\frac{2 \lambda}{1+\lambda} \hat{x}_{12}^{a}=\sum_{p=0}^{\infty} \sum_{q=0}^{\infty} U_{p q} t_{1}^{p} t_{2}^{q} .
$$

Then, we consider the problem in which the forces are given by

$$
\left(6 \pi \eta a_{1}\right)^{-1} \mathbf{F}_{1}=\left(6 \pi \eta a_{2}\right)^{-1} \mathbf{F}_{2}=U \mathbf{e} .
$$

It is easy to find that the velocities $U_{x}$ in this problem can be expanded in the form of eq. (57) with coefficients $U_{p q}$ being replaced by $(-1)^{p+a} U_{p q}$. Thus, a relation between the mobility functions in the form similar to eq. (60) can be obtained.

As in the case of resistance functions, the mobility functions $\hat{x}_{11}^{a}$ and $\hat{x}_{12}^{a}$ are given by a series either of even powers of $s^{-1}$ or of odd powers,

$$
\begin{aligned}
& \hat{x}_{11}^{a}=\sum_{k=0}^{\infty} f_{2 k}(\hat{\lambda})(1+\lambda)^{-2 k} s^{-2 k} \\
& \hat{x}_{12}^{a}=-\frac{1}{2} \sum_{k=0}^{\infty} f_{2 k+1}(\lambda)(1+\lambda)^{-2 k} s^{-2 k-1}
\end{aligned}
$$

where

$f_{k}(\lambda)=2^{k} \sum_{q=0}^{k} U_{(k-q) q} \lambda^{q-j}, \quad j= \begin{cases}0 & \text { if } k \text { is even } \\ 1 & \text { if } k \text { is odd }\end{cases}$

Explicitly,

$$
\begin{aligned}
& f_{0}=\beta_{32}, \quad f_{1}=-3, \quad f_{2}=0 \\
& f_{3}=4 \beta_{02}\left(1+\lambda^{2}\right), \quad f_{4}=-60 \beta_{25} \lambda^{3}, \quad f_{5}=0 \\
& f_{6}=480 \beta_{05} \hat{\lambda}^{3}-\frac{32}{5}\left(2 \beta_{(-3) 2}-45 \beta_{05}+63 \beta_{27}\right) \lambda^{5} \\
& f_{7}=-2400 \beta_{25}^{2} \lambda^{3} \\
& \text { If } \hat{\beta}=0 \\
& f_{8}=-384 \lambda^{7}, \quad f_{9}=-2304\left(\lambda^{3}+\lambda^{5}\right) \\
& f_{10}=-1536\left(4 \lambda^{6}+\lambda^{9}\right) \\
& f_{11}=-6144\left(2 \lambda^{3}+3 \lambda^{5}+2 \lambda^{7}\right) .
\end{aligned}
$$


The expressions of $f_{8}, f_{9}, f_{10}$ and $f_{11}$ for the limiting situation of no-slip spheres $(\hat{\beta} \rightarrow \infty)$ can also be found in Jeffrey and Onishi (1984).

Using the so-called connector algebra, Geigenmuller and Mazur (1986) obtained the explicit formulas for the mobility functions $\hat{x}_{11}^{a}, \hat{x}_{12}^{a}, \hat{y}_{11}^{a}$ and $\hat{y}_{12}^{a}$ in power series of $1 / r$ up to $\mathrm{O}\left(r^{-7}\right)$ for the case of two identical fluid drops. The above expressions agree with (and are more accurate than) their results (taking the viscosity of the drops as zero) for the case when $\widehat{\beta}=0$. Again, the interaction between two slip spheres with a finite value of $\hat{\beta}$ can be found to differ from that between two fluid drops with a value of $3 \eta^{*}$ equal to $\hat{\beta}$.

The exact (numerical) solution of the mobility functions $\hat{x}_{11}^{a}$ and $\hat{x}_{12}^{a}$ for two slip spheres was also obtained by using bipolar coordinates (Chen and Keh, 1995). Table 2 gives a comparison of our asymptotic results with the exact solution for the case of two

Table 2. The normalized velocities $U / U^{(0)}$ of two identical spheres exerted by equal forces along the line of their centers

\begin{tabular}{|c|c|c|c|c|}
\hline \multirow{2}{*}{$\frac{\beta a}{\eta}$} & \multirow{2}{*}{$\frac{2 a}{r}$} & \multicolumn{2}{|c|}{ Asymptotic solution } & \multirow{2}{*}{$\begin{array}{c}\text { Exact } \\
\text { solution }\end{array}$} \\
\hline & & $O\left(s^{-7}\right)$ & $O\left(s^{-11}\right)$ & \\
\hline \multirow[t]{9}{*}{0} & 0.2 & 1.0999 & 1.0999 & 1.0999 \\
\hline & 0.4 & 1.1984 & 1.1984 & 1.1984 \\
\hline & 0.6 & 1.2916 & 1.2917 & 1.2917 \\
\hline & 0.8 & 1.3736 & 1.3746 & 1.3743 \\
\hline & 0.9 & 1.4082 & 1.4115 & 1.4103 \\
\hline & 0.95 & 1.4235 & 1.4293 & 1.4269 \\
\hline & 0.99 & 1.4348 & 1.4436 & 1.4396 \\
\hline & 0.999 & 1.4372 & 1.4469 & 1.4424 \\
\hline & 0.9999 & 1.4375 & 1.4472 & 1.4427 \\
\hline \multirow[t]{9}{*}{1} & 0.2 & 1.1121 & & 1.1121 \\
\hline & 0.4 & 1.2208 & & 1.2208 \\
\hline & 0.6 & 1.3200 & & 1.3201 \\
\hline & 0.8 & 1.4031 & & 1.4040 \\
\hline & 0.9 & 1.4375 & & 1.4395 \\
\hline & 0.95 & 1.4532 & & 1.4558 \\
\hline & 0.99 & 1.4652 & & 1.4682 \\
\hline & 0.999 & 1.4678 & & 1.4709 \\
\hline & 0.9999 & 1.4681 & & 1.4712 \\
\hline \multirow[t]{9}{*}{10} & 0.2 & 1.1374 & & 1.1374 \\
\hline & 0.4 & 1,2667 & & 1.2667 \\
\hline & 0.6 & 1.3769 & & 1.3770 \\
\hline & 0.8 & 1.4649 & & 1.4624 \\
\hline & 0.9 & 1.5072 & & 1.4970 \\
\hline & 0.95 & 1.5311 & & 1.5127 \\
\hline & 0.99 & 1.5532 & & 1.5245 \\
\hline & 0.999 & 1.5586 & & 1.5271 \\
\hline & 0.9999 & 1.5591 & & 1.5274 \\
\hline \multirow[t]{9}{*}{$\infty$} & 0.2 & 1.1486 & 1.1486 & 1.1486 \\
\hline & 0.4 & 1.2866 & 1.2866 & 1.2866 \\
\hline & 0.6 & 1.4007 & 1.4006 & 1.4006 \\
\hline & 0.8 & 1.4932 & 1.4853 & 1.4861 \\
\hline & 0.9 & 1.5458 & 1.5143 & 1.5202 \\
\hline & 0.95 & 1.5799 & 1.5218 & 1.5356 \\
\hline & 0.99 & 1.6135 & 1.5213 & 1.5472 \\
\hline & 0.999 & 1.6221 & 1.5201 & 1.5497 \\
\hline & 0.9999 & 1.6229 & 1.5199 & 1.5500 \\
\hline
\end{tabular}

identical spheres experiencing equal applied forces $\left(F_{1}=F_{2}=F e\right)$. In this case, the particles will translate at the same velocity $\left(U_{1}=U_{2}=U\right)$. It is found in Table 2 that the predictions of $U / U^{(0)}$ $\left[=\left(\hat{x}_{11}^{a}+\hat{x}_{12}^{a}\right)(\beta a+3 \eta) /(\beta a+2 \eta)\right.$, where $U^{(0)}$ is the translational velocity of each particle subject to the applied force in the absence of the other particle] from the asymptotic approximation for various values of $\beta a / \eta$ are in perfect agreement with those of the exact solution. The errors in velocities are less than $1.7 \%$ for cases $2 a / r \leqslant 0.9$ or $4.7 \%$ for cases $2 a / r \leqslant 0.9999$ if the asymptotic formula (62) is accurate to $O\left(s^{-7}\right)$. For the situation of two spheres differing in size, eq. (62) can also be found to agree very well with the exact solution. Similar to the resistance problem considered in Section 4.1, the error of eq. (62) with finite terms is a monotonic increasing function of $\beta a / \eta$ for a given value of $s$. However, the results obtained from the method of twin multipole expansions for the mobility problem are much more accurate (and simpler in expression) than those (with the same order of $s^{-1}$ in the expansions) for the resistance problem. Thus, the better way to evaluate the resistance functions through series expansions in $s^{-1}$ is to calculate the mobility functions first and then use the reciprocal relations to determine the resistance functions.

\section{2. $\hat{\mathrm{y}}_{\alpha \beta}^{\mathrm{a}}(\mathrm{s}, \lambda)$}

We now consider the motion of two spheres perpendicular to the line of their centers, and again define two problems. First

$$
\left(6 \pi \eta a_{1}\right)^{-1} \mathbf{F}_{1}=-\left(6 \pi \eta a_{2}\right)^{-1} \mathbf{F}_{2}=U \mathbf{i}
$$

and, secondly,

$$
\left(6 \pi \eta a_{1}\right)^{-1} \mathbf{F}_{1}=\left(6 \pi \eta a_{2}\right)^{-1} \mathbf{F}_{2}=U \mathbf{i}
$$

with in each case $\mathbf{T}_{1}=\mathbf{T}_{2}=\mathbf{0}$. The purpose is to find the translational velocities $\mathbf{U}_{1}=U_{1} \mathbf{i}$ and $\mathbf{U}_{2}=$ $\mp U_{2} \mathbf{i}$ as well as the angular velocities $\boldsymbol{\Omega}_{1}=-\boldsymbol{\Omega}_{\mathbf{1}} \mathbf{j}$ and $\mathbf{\Omega}_{2}=\mp \Omega_{2} \mathbf{j}$.

For the first problem, the coefficients defined in eq. (19) become

$$
\begin{gathered}
\chi_{m n}^{(\alpha)}=(-1)^{3-\alpha} U_{\alpha} \delta_{m 1} \delta_{n 1}, \quad \psi_{m n}^{(\alpha)}=0 \\
\omega_{m n}^{(\alpha)}=2 a_{\alpha} \Omega_{\alpha} \delta_{m 1} \delta_{n 1} .
\end{gathered}
$$

The quantities $p_{1 n}^{(\alpha)}, q_{1 n}^{(\alpha)}, v_{1 n}^{(\alpha)}, U_{\alpha}$ and $\Omega_{\alpha}$ can be expanded as

$$
\begin{aligned}
p_{1 n}^{(\alpha)} & =(-1)^{3-\alpha} \frac{3}{2} U \sum_{p=0}^{\infty} \sum_{q=0}^{\infty} P_{n p q} t_{\alpha}^{p} t_{3-\alpha}^{q} \\
q_{1 n}^{(\alpha)} & =\mathrm{i} U \sum_{p=0}^{\infty} \sum_{q=0}^{\infty} Q_{n p q} t_{\alpha}^{p} t_{3-\alpha}^{q} \\
v_{1 n}^{(\alpha)} & =(-1)^{3-\alpha} \frac{3}{4} U \sum_{p=0}^{\infty} \sum_{q=0}^{\infty} \frac{1}{2 n+1} V_{n p q} t_{\alpha}^{p} t_{3-\alpha}^{q} \\
U_{\alpha} & =(-1)^{3-\alpha} U \sum_{p=0}^{\infty} \sum_{q=0}^{\infty} U_{p q} t_{\alpha}^{p} t_{3-\alpha}^{q} \\
a_{\alpha} \Omega_{\alpha} & =U \sum_{p=0}^{\infty} \sum_{q=0}^{\infty} \Omega_{p q} t_{\alpha}^{p} t_{3-\alpha}^{q} .
\end{aligned}
$$


Using eqs (21), (64) and (67a,b), one finds

$$
P_{1 p q}=\delta_{p 0} \delta_{q 0}, \quad Q_{1 p q}=0 .
$$

All coefficients $V_{n p q}$ are calculated from the relation

$$
\begin{aligned}
V_{n p q}= & \frac{2 n+1}{(n+1)\left[1+(2 n+1) \hat{\beta}^{-1}\right]} \sum_{s=1}^{q}\left(\begin{array}{l}
n+s \\
n+1
\end{array}\right) \\
& \times\left\{\frac{(n+s)(n s+4)-2(n s+1)^{2}}{2 s(2 s-1)(n+s)}\right. \\
& \times P_{s(q-s)(p-n+1)} \\
& +\beta_{(-2) 0} \frac{n(2 n+1)}{2(2 n+3)} P_{s(q-s)(p-n-1)} \\
& -\frac{2}{3}(2 n-1) Q_{s(q-s-1)(p-n+1)} \\
& \left.+\frac{n(2 n-1)}{2(2 s+1)} V_{s(q-s-2)(p-n+1)}\right\}+\delta_{n 1} \beta_{03} U_{p q} .
\end{aligned}
$$

For $n>1$, the recurrence relations for coefficients $P_{n p q}$ and $Q_{n p q}$ are given by eqs $(38 \mathrm{~b}, \mathrm{c})$. Substitution of $n=1$ into eqs $(20 \mathrm{~b}, \mathrm{c})$ using eqs $(66)$ and $(67)$ gives expressions for coefficients $U_{p q}$ and $\Omega_{p q}$ :

$$
\begin{aligned}
U_{p q}= & \sum_{s=1}^{q} \frac{s(s+1)}{2}\left\{\frac{3(s-2)}{4 s(2 s-1)} P_{s(q-s) p}\right. \\
& -\frac{1}{4} \beta_{02} P_{s(q-s)(p-2)}+Q_{s(q-s-1) p} \\
& \left.-\frac{3}{4(2 s+1)} V_{s(q-s-2) p}\right\} \\
\Omega_{p q}= & \sum_{s=1}^{q} \frac{s(s+1)}{4}\left\{\frac{3}{2 s} P_{s(q-s)(p-1)}-s Q_{s(q-s-1)(p-1)}\right\} .
\end{aligned}
$$

The coefficients $U_{p q}$ are related to the mobility functions by

$$
\hat{y}_{11}^{a}-\frac{2}{1+\lambda} \hat{y}_{12}^{a}=\sum_{p=0}^{\infty} \sum_{q=0}^{\infty}(-1)^{p+q} U_{p q} t_{1}^{p} t_{2}^{q} .
$$

When the second problem is considered and its result is compared with eq. (73), one has

$$
\begin{aligned}
& \hat{y}_{11}^{a}=\sum_{k=0}^{\infty} f_{2 k}(\lambda)(1+\lambda)^{-2 k} s^{-2 k} \\
& \hat{y}_{12}^{a}=\frac{1}{2} \sum_{k=0}^{\infty} f_{2 k+1}(\lambda)(1+\lambda)^{-2 k} s^{-2 k-1}
\end{aligned}
$$

where $f_{k}(\lambda)$ is given by eq. (63) and the first few values are

$$
\begin{aligned}
& f_{0}=\beta_{32}, \quad f_{1}=\frac{3}{2}, \quad f_{2}=0 \\
& f_{3}=2 \beta_{02}\left(1+\lambda^{2}\right), \quad f_{4}=f_{5}=0 \\
& f_{6}=-\frac{4}{5}\left(4 \beta_{(-3) 2}+60 \beta_{(-1) 4}+21 \beta_{27}\right) \lambda^{5}, \quad f_{7}=0 . \\
& \text { If } \hat{\beta}=0, \\
& f_{8}=\frac{384}{5} \lambda^{7}, \quad f_{9}=0, \quad f_{10}=384 \lambda^{9}, \quad f_{11}=576 \lambda^{5} .
\end{aligned}
$$

Again, these formulas are consistent with and more accurate than the results obtained by Geigenmuller and Mazur (1986) for the motion of two identical fluid drops (taking $\eta^{*}=0$ ) in the limit $\hat{\beta}=0$. Similar to the resistance problem, the expressions of $\hat{x}_{11}^{A}, \hat{x}_{12}^{A}$, $\hat{y}_{11}^{A}$ and $\hat{y}_{12}^{A}$ for the motion of two slip spheres and for the motion of two spherical drops at finite values of $\hat{\beta}$ and $\eta^{*}$ are identical up to $O\left(r^{-3}\right)$ if one puts $\widehat{\beta}=3 \eta^{*}$; the difference between them appears when terms of $O\left(r^{-4}\right)$ are included.

\section{3. $\hat{\mathrm{y}}_{\alpha \beta}^{\mathrm{b}}(\mathrm{s}, \lambda)$}

The calculations in the preceding subsection can be used to find functions $\hat{y}_{\alpha \beta}^{b}(s, \lambda)$. The coefficients $\Omega_{p q}$ in series expansion (69) for the angular velocity of a sphere translating under an applied force are given by eq. (72). The functions $\hat{y}_{\alpha \beta}^{b}$ are then deduced in the standard way with the difference that odd powers of $s^{-1}$ in the series go to $\hat{y}_{11}^{b}$ and even powers to $\hat{y}_{12}^{b}$. The result is

$$
\begin{aligned}
& \hat{y}_{11}^{b}=\sum_{k=0}^{\infty} f_{2 k+1}(\lambda)(1+\lambda)^{-2 k-1} s^{-2 k-1} \\
& \hat{y}_{12}^{b}=\frac{1}{4} \sum_{k=0}^{\infty} f_{2 k}(\lambda)(1+\lambda)^{-2 k+2} s^{-2 k}
\end{aligned}
$$

Here

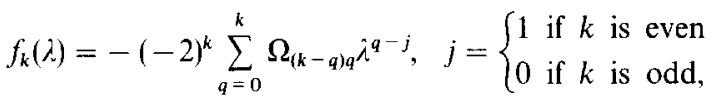

and some explicit expressions are

$$
\begin{aligned}
& f_{0}=f_{1}=0, \quad f_{2}=-2, \quad f_{3}=f_{4}=f_{5}=f_{6}=0 \\
& f_{7}=160 \beta_{05} \lambda^{3}+16\left(6 \beta_{05}+4 \beta_{(-1) 4}-7 \beta_{27}\right) \lambda^{5} .
\end{aligned}
$$

\section{4. $\hat{\mathrm{x}}_{x \beta}^{\mathrm{c}}\left(\mathrm{s}, \lambda_{\mathrm{n}}\right)$}

In order to determine the functions $\hat{x}_{\alpha \beta}^{c}(s, \lambda)$, we consider the torques acting on the two spheres given by

$$
\left(8 \pi \eta a_{1}^{3}\right)^{-1} \mathbf{T}_{1}=\mp\left(8 \pi \eta a_{2}^{3}\right)^{-1} \mathbf{T}_{2}=\Omega \mathbf{e} .
$$

Because the translational velocities are zero (given $\mathbf{F}_{1}=\mathbf{F}_{2}=\mathbf{0}$ ), the only non-zero functions in eq. (20) are $q_{0 n}^{(\alpha)}$. When the minus sign in eq. (77) is considered, $q_{0 n}^{(\alpha)}$ can be expanded as eq. (46) and the particle angular velocities can be expressed as eq. (69) with $U$ being replaced by $a_{x} \Omega$ now. By the combination of eqs (20c), (21b) and (69), the coefficients $\Omega_{p q}$ are obtained from the relation

$$
\Omega_{p q}=\beta_{30} Q_{1 p q}-\sum_{s=0}^{q} \frac{s(s+1)}{2} Q_{s(q-s-1)(p \cdots 1)} .
$$

The recurrence relation for coefficients $Q_{n p q}$ is given by eq. (47b) with the initial value $Q_{n 00}=\delta_{n 1}$. Using eqs (21b), (46), (69) and (78) and observing that the even and odd powers of $s^{-1}$ in the series go to the functions $\hat{x}_{11}^{c}$ and $\hat{x}_{12}^{c}$, respectively, one 
obtains

$$
\begin{aligned}
& \hat{x}_{11}^{c}=\sum_{k=0}^{\infty} f_{2 k}(\lambda)(1+\lambda)^{-2 k} s^{-2 k} \\
& \hat{x}_{12}^{c}=-\frac{1}{8} \sum_{k=0}^{\infty} f_{2 k+1}(\lambda)(1+\lambda)^{-2 k+2} s^{-2 k-1}
\end{aligned}
$$

where

$f_{k}(\lambda)=\sum_{q=0}^{k} \Omega_{(k-q) q} \lambda^{q-j}, j=\left\{\begin{array}{l}0 \text { if } k \text { is even } \\ 2 \text { if } k \text { is odd }\end{array}\right.$

Explicitly,

$$
\begin{aligned}
& f_{0}=\beta_{30}, \quad f_{1}=f_{2}=0 \\
& f_{3}=1, \quad f_{4}=f_{5}=f_{6}=f_{7}=0 .
\end{aligned}
$$

Note that $\hat{x}_{11}^{c} \rightarrow \infty$, as expected, and $\hat{x}_{12}^{c}=-\frac{1}{8} s^{-3}$ in the limit $\hat{\beta}=0$.

\section{5. $\hat{y}_{\alpha \beta}^{\mathrm{c}}(\mathrm{s}, \lambda)$}

The functions $\hat{y}_{\alpha \beta}^{c}(s, \lambda)$ are to be obtained from problems of two spheres in the situation that

$$
\left(8 \pi \eta a_{1}^{3}\right)^{-1} \mathbf{T}_{1}= \pm\left(8 \pi \eta a_{2}^{3}\right)^{-1} \mathbf{T}_{2}=\Omega \mathbf{i}
$$

with $\mathbf{F}_{1}=\mathbf{F}_{2}=\mathbf{0}$. The recurrence relations used to calculate $\hat{y}_{\alpha \beta}^{a}$ in Section 5.2 with $U$ being replaced by $a_{\alpha} \Omega$ can be used here with only a change of initial conditions,

$$
P_{1 p q}=0, \quad Q_{1 p q}=\delta_{p 0} \delta_{q 0}, \quad V_{1 p q}=0 .
$$

As usual, we have

$$
\begin{aligned}
& \hat{y}_{11}^{c}=\sum_{k=0}^{\infty} f_{2 k}(\lambda)(1+\lambda)^{-2 k} s^{-2 k} \\
& \hat{y}_{12}^{c}=\frac{1}{8} \sum_{k=0}^{\infty} f_{2 k+1}(\lambda)(1+\lambda)^{-2 k+2} s^{-2 k-1} .
\end{aligned}
$$

Here, $f_{k}(\lambda)$ is given by the form of eq. (80), and the first few values are

$$
\begin{gathered}
f_{0}=\beta_{30}, \quad f_{1}=f_{2}=0, \quad f_{3}=-4, \quad f_{4}=f_{5}=0 \\
f_{6}=-24\left(\beta_{03}+9 \beta_{25}\right) \lambda^{3}, \quad f_{7}=0 .
\end{gathered}
$$

As expected, $\hat{y}_{11}^{c} \rightarrow \infty$ in the limit $\hat{\beta}=0$.

\section{AVERAGE SETTLING VELOCITY IN A SUSPENSION OF SLIP SPHERES}

In practical applications of sedimentation phenomena, collections of particles in bounded systems are usually encountered. The interaction effects between pairs of spheres discussed in the previous section can be used to find how the average settling (or buoyantly rising) velocity of a dilute suspension of slip spheres is affected by the volume fraction of the particles. Based on a microscopic model of particle interactions in a dilute dispersion which comprises both statistical and low Reynolds number hydrodynamic concepts (Batchelor, 1972; Reed and Anderson, 1980), the average settling velocity of a test particle (with radius $a_{t}$ ), which samples all positions in a bounded dispersion, is given by

$$
\begin{aligned}
\left\langle\mathbf{U}_{t}\right\rangle= & \mathbf{U}_{t}^{(0)}+C\left\{\int_{V} \mathbf{v}^{*}(\mathbf{r})[g(\mathbf{r})-1] \mathrm{d} \mathbf{r}\right. \\
& +\frac{a_{t}^{2}}{6 A_{2}} \int_{r>\mathbf{a}} \nabla^{2} \mathbf{v}^{*}(\mathbf{r})[g(\mathbf{r})-1] \mathrm{d} \mathbf{r} \\
& +\frac{4}{3} \pi a_{t}^{2} a\left(\frac{3}{2} \beta_{23}-1\right) \mathbf{U}^{(0)} \\
& \left.+\int_{V} \mathbf{W}(\mathbf{r}) g(\mathbf{r}) \mathrm{d} \mathbf{r}\right\}+O\left(C^{2}\right) .
\end{aligned}
$$

Here, $C$ is the macroscopic concentration of the neighboring particles (assumed to be identical, with radius $a), \mathbf{U}_{t}^{(0)}$ and $\mathbf{U}^{(0)}$ are the undisturbed settling velocities of the test particle and a neighboring particle, respectively, $g(\mathbf{r})$ is the two-particle radial distribution function, and $V$ denotes the entire volume of the dispersion. $\mathbf{v}^{*}(\mathbf{r})$ is the velocity field at position $\mathbf{r}$ when a single neighbor particle at the origin $\mathbf{0}$ moves with velocity $\mathbf{U}^{(0)}$, which can be expressed as (Basset, 1961)

$$
\begin{aligned}
& r<a: \quad \mathbf{v}^{*}(\mathbf{r})=\mathbf{U}^{(0)} \\
& r>a: \quad \mathbf{v}^{*}(\mathbf{r})= {\left[\frac{3}{4} \beta_{23}\left(\frac{a}{r}\right)+\frac{1}{4} \beta_{03}\left(\frac{a}{r}\right)^{3}\right](\mathbf{I}-\mathbf{e e}) \cdot \mathbf{U}^{(0)} } \\
&+\left[\frac{3}{2} \beta_{23}\left(\frac{a}{r}\right)-\frac{1}{2} \beta_{03}\left(\frac{a}{r}\right)^{3}\right] \mathbf{e e} \cdot \mathbf{U}^{(0)}
\end{aligned}
$$

The Laplacian of this field can be found to be

$$
\begin{gathered}
r<a: \quad \nabla^{2} \mathbf{v}^{*}(\mathbf{r})=\mathbf{0}, \\
r>a: \quad \nabla^{2} \mathbf{v}^{*}(\mathbf{r})=\frac{3}{2} \beta_{23}\left(\frac{a}{r^{3}}\right)[\mathbf{l}-3 \mathbf{e e}] \cdot \mathbf{U}^{(0)}
\end{gathered}
$$

$\mathbf{W}(\mathbf{r})$ in eq. (84) is a correction function needed to account for the perturbation on $\mathbf{v}^{*}$ owing to the presence of the test particle and the boundary, and is given by

$$
\mathbf{W}(\mathbf{r})=\mathbf{U}_{\mathbf{t}}^{*}(\mathbf{r})-\mathbf{U}_{t}^{(0)}-\mathbf{v}^{*}(\mathbf{r})-\frac{1}{6} \beta_{02} a_{t}^{2} \nabla^{2} \mathbf{v}^{*}(\mathbf{r})
$$

where $\mathbf{U}_{t}^{*}(\mathbf{r})$ is the actual velocity of the test particle located at $\mathbf{r}$ with respect to the origin of a single neighbor at $\mathbf{0}$. If all the particles have the same value of $\hat{\beta}, \mathbf{U}_{t}^{*}(\mathbf{r})$ can be calculated using the mobility functions derived in Sections 5.1 and 5.2,

$$
\begin{aligned}
\mathbf{U}_{t}^{*}(\mathbf{r})= & {\left[\hat{x}_{11}^{a} \mathbf{U}_{t}^{(0)}+\frac{2 \lambda}{1+\lambda} \hat{x}_{12}^{a} \mathbf{U}^{(0)}\right] \cdot \text { ee } } \\
& +\left[\hat{y}_{11}^{a} \mathbf{U}_{t}^{(0)}+\frac{2}{1+\lambda} \hat{y}_{12}^{a} \mathbf{U}^{(0)}\right] \cdot(\mathbf{I}-\mathbf{e e})
\end{aligned}
$$

where subscripts 1 and 2 denote the test and neighboring particles, respectively. Substitution of eqs (85b), (86b) and (88) into eq. (87) shows that the magnitude of $\mathbf{W}$ behaves as $r^{-4}$ when $r \gg a$.

The volume integrals in eq. (84) can be evaluated by assuming that the radial distribution function has the 
following equilibrium value for rigid spheres without long-range pair potential:

$$
\begin{array}{ll}
g=0 & \text { if } r<a_{t}+a \\
g=1+O(C) & \text { if } r>a_{t}+a
\end{array}
$$

where $O(C)$ is a term proportional to the concentration of the neighbor particles. That is, the particles must be sufficiently small so that Brownian motion dominates any multiparticle hydrodynamic interactions which might impart microscopic structure to the dispersion. In general, it is necessary to obtain the pair distribution function as the solution of a conservation equation of the Fokker-Planck type for a polydisperse system of spheres (Batchelor, 1982). The conditions under which the assumption of local equilibrium is valid for a dilute dispersion comprising different types of particles are also discussed by Reed and Anderson (1980).

Given eqs $(85)-(89)$ and the relation $\mathbf{U}^{(0)}$ $=\mathbf{U}_{t}^{(0)} a^{2} / a_{t}^{2}$ (assuming that the test particle and its neighbors have the same density), the integrals in eq. (84) can be evaluated to yield

$$
\left\langle\mathbf{U}_{t}\right\rangle=\mathbf{U}_{t}^{(0)}\left[1+x_{t} \varphi+O\left(\varphi^{2}\right)\right]
$$

with

$$
\begin{aligned}
\alpha_{t}= & -\left[1+3 \beta_{23}\left(\frac{a}{a_{t}}\right)+\left(\frac{a}{a_{t}}\right)^{2}\right] \\
& -\frac{15}{4} \beta_{23} \beta_{25}\left(\frac{a}{a_{t}+a}\right)+\frac{5}{2} \beta_{03} \beta_{25}\left(\frac{a}{a_{t}+a}\right)^{3} \\
& -\frac{1}{40} \beta_{23}\left(4 \beta_{(-3) 2}+20 \beta_{(-1) 4}-60 \beta_{05}\right. \\
& \left.+91 \beta_{27}\right) \frac{a_{t} a^{2}}{\left(a_{t}+a\right)^{3}}+\frac{75}{16} \beta_{23} \beta_{25}^{2} \frac{a_{t} a^{3}}{\left(a_{t}+a\right)^{4}}
\end{aligned}
$$

where $\varphi\left(=4 \pi a^{3} C / 3\right)$ is the volume fraction of the neighbor particles. Note that the integral of the modified Faxen correction involving $\nabla^{2} \mathbf{v}^{*}$ (Felderhof, $1976)$ in eq. (84) equals zero as computed from eqs $(86 \mathrm{~b})$ and (89). The term inside the brackets in eq. (91) for $\alpha_{1}$ is obtained from the first and third terms in the braces of eq. (84), while the other terms in eq. (91) are the result of the last integral in eq. (84). Certainly, this expression is not exact, even given that eq. (89) holds, because $O\left(r^{-8}\right)$ terms are neglected in the evaluation of $\mathbf{U}_{t}^{*}$; however, the error should be small and will appear only in the calculation involving the correction function $\mathbf{W}$. In the derivation of eq. (91), all the neighboring particles are assumed to be identical, even though they are allowed to differ in radius from the test particle.

For a dispersion of particles that have a distribution in radius, a generalization of eqs (90) and (91) leads to

$$
\left\langle\mathbf{U}_{i}\right\rangle=\mathbf{U}_{i}^{(0)}\left[1+\sum_{j} \alpha_{i j} \varphi_{j}+O\left(\varphi^{2}\right)\right]
$$

where

$$
\begin{aligned}
\alpha_{i j}= & -\left[1+3 \beta_{23}\left(\frac{a_{j}}{a_{i}}\right)+\left(\frac{a_{j}}{a_{i}}\right)^{2}\right] \\
& -\frac{15}{4} \beta_{23} \beta_{25}\left(\frac{a_{i}}{a_{i}+a_{j}}\right)+\frac{5}{2} \beta_{03} \beta_{25}\left(\frac{a_{i}}{a_{i}+a_{j}}\right)^{3} \\
& -\frac{1}{40} \beta_{23}\left(4 \beta_{(-3) 2}+20 \beta_{(-1) 4}-60 \beta_{05}\right. \\
& \left.+91 \beta_{27}\right) \frac{a_{i} a_{j}^{2}}{\left(a_{i}+a_{j}\right)^{3}}+\frac{75}{16} \beta_{23} \beta_{25}^{2} \frac{a_{i} a_{j}^{3}}{\left(a_{i}+a_{j}\right)^{4}}
\end{aligned}
$$

Here, subscript $i$ denotes the type of particles having radius $a_{i}$. Note that eqs (91) and (93) are valid only when all the particles have the same density and value of $\hat{\beta}$.

An examination of eq. (93) indicates that the interaction coefficient $\alpha_{i j}$ is always negative, irrespective of values of $a_{j} / a_{i}$ and $\hat{\beta}$. Thus, the average settling velocity of a type of particles is reduced with an increase in the concentration of any type of particles in the suspension. Results of $\alpha_{i j}$ calculated from eq. (93) at various values of $a_{j} / a_{i}$ are plotted vs $\hat{\beta}$ in Fig. 1 . It can be seen that, as expected, the magnitude of $\alpha_{i j}$, which reflects the intensity of particle interactions, increases monotonically with the increase of $\hat{\beta}$ for a fixed value of $a_{j} / a_{i}$. If the value of $\hat{\beta}$ is kept constant, the magnitude of $\alpha_{i j}$ is a monotonic increasing function of the ratio $a_{j} / a_{i}$. Namely, the influence of the interactions on the smaller particles is stronger than on the larger ones. Note that, in the limit $a_{j} / a_{i}=0$, eq. (93) predicts that $\alpha_{i j}=-2$ for the case of $\hat{\beta}=0$ and $\alpha_{i j}=-\frac{9}{4}$ [if more accurate values of the mobility functions in eq. (88) are used, $\alpha_{i j}=-\frac{7}{2}$ as indicated by eq. (94)] for the case of $\hat{\beta} \rightarrow \infty$.

For a suspension of no-slip spheres $(\hat{\beta} \rightarrow \infty)$, the mobility functions in eq. (88) can be calculated to the order of $r^{-11}$ (Jeffrey and Onishi, 1984); thus, a more accurate expression for $\alpha_{i j}$ than eq. (93) is obtained,

$$
\begin{aligned}
\alpha_{i j}= & -\left[1+3 \frac{a_{j}}{a_{i}}+\left(\frac{a_{j}}{a_{i}}\right)^{2}\right]-\frac{15}{4}\left(\frac{a_{i}}{a_{i}+a_{j}}\right) \\
& +\frac{5}{2}\left(\frac{a_{i}}{a_{i}+a_{j}}\right)^{3}-\frac{11}{8} \frac{a_{i} a_{j}^{2}}{\left(a_{i}+a_{j}\right)^{3}}+\frac{75}{16} \frac{a_{i} a_{j}^{3}}{\left(a_{i}+a_{j}\right)^{4}} \\
& -\frac{5}{4}\left(\frac{a_{i}}{a_{i}+a_{j}}\right)^{5}+\frac{15}{4} \frac{a_{i}^{3} a_{j}^{2}}{\left(a_{i}+a_{j}\right)^{5}}-\frac{9}{10} \frac{a_{i} a_{j}^{4}}{\left(a_{i}+a_{j}\right)^{5}} \\
& -\frac{5}{8} \frac{a_{i}^{3} a_{j}^{3}}{\left(a_{i}+a_{j}\right)^{6}}-\frac{5}{8} \frac{a_{i} a_{j}^{5}}{\left(a_{i}+a_{j}\right)^{6}}-\frac{35}{8} \frac{a_{i}^{5} a_{j}^{2}}{\left(a_{i}+a_{j}\right)^{7}} \\
& -\frac{375}{28} \frac{a_{i}^{4} a_{j}^{3}}{\left(a_{i}+a_{j}\right)^{7}}+\frac{21}{4} \frac{a_{i}^{3} a_{j}^{4}}{\left(a_{i}+a_{j}\right)^{7}}-\frac{9}{14} \frac{a_{i} a_{j}^{6}}{\left(a_{i}+a_{j}\right)^{7}} \\
& +\frac{25}{8} \frac{a_{i}^{5} a_{j}^{3}}{\left(a_{i}+a_{j}\right)^{8}}-\frac{4177}{256} \frac{a_{i}^{3} a_{j}^{5}}{\left(a_{i}+a_{j}\right)^{8}} \\
& +\frac{25}{8} \frac{a_{i} a_{j}^{7}}{\left(a_{i}+a_{j}\right)^{8}}
\end{aligned}
$$




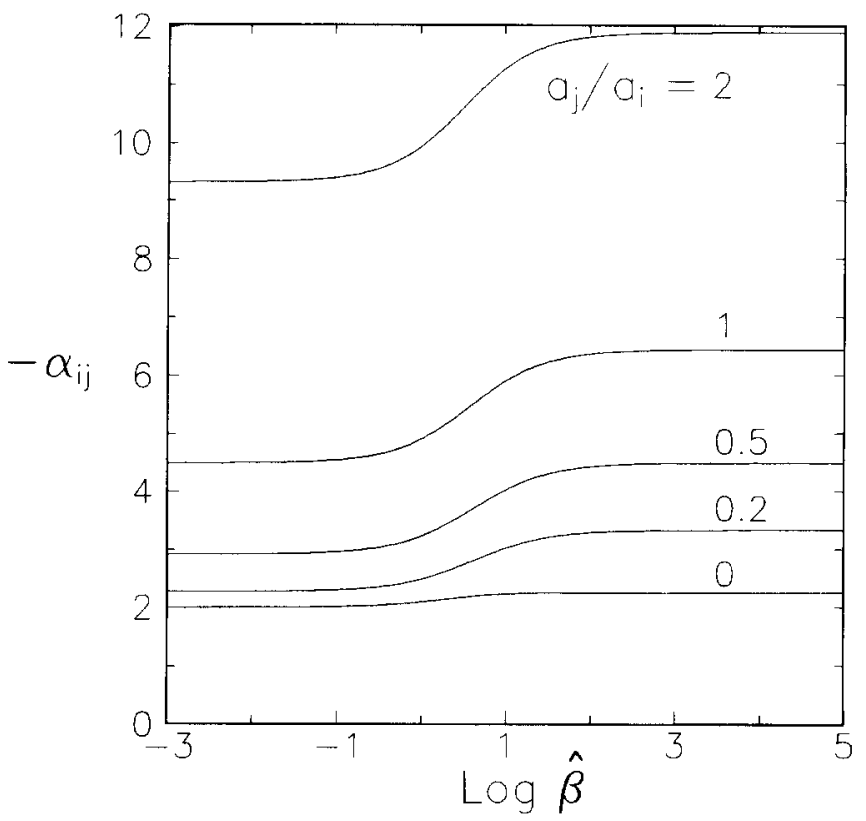

Fig. 1. Plots of coefficient $\alpha_{i j}$ calculated from eq. (93) for a bounded suspension of slip spheres vs $\hat{\beta}$ with $a_{j} / a_{i}$ as a parameter.

Similarly, for a suspension of perfect-slip spheres or spherical gas bubbles $(\hat{\beta}=0), \alpha_{i j}$ can be evaluated by the following formula of the same accuracy:

$$
\begin{aligned}
\alpha_{i j}= & -\left[1+2 \frac{a_{j}}{a_{i}}+\left(\frac{a_{j}}{a_{i}}\right)^{2}\right]-\frac{a_{i}}{a_{i}+a_{j}}-\frac{1}{4} \frac{a_{i} a_{j}^{2}}{\left(a_{i}+a_{j}\right)^{3}} \\
& +\frac{1}{2} \frac{a_{i} a_{j}^{3}}{\left(a_{i}+a_{j}\right)^{4}}-\frac{3}{25} \frac{a_{i} a_{j}^{4}}{\left(a_{i}+a_{j}\right)^{5}}+\frac{1}{2} \frac{a_{i}^{3} a_{j}^{3}}{\left(a_{i}+a_{j}\right)^{6}} \\
& +\frac{1}{2} \frac{a_{i} a_{j}^{5}}{\left(a_{i}+a_{j}\right)^{6}}-\frac{4}{7} \frac{a_{i}^{4} a_{j}^{3}}{\left(a_{i}+a_{j}\right)^{7}}-\frac{1}{14} \frac{a_{i} a_{j}^{6}}{\left(a_{i}+a_{j}\right)^{7}} \\
& +\frac{1}{2} \frac{a_{i}^{5} a_{j}^{3}}{\left(a_{i}+a_{j}\right)^{8}}+\frac{51}{64} \frac{a_{i}^{3} a_{j}^{5}}{\left(a_{i}+a_{j}\right)^{8}}+\frac{1}{2} \frac{a_{i} a_{j}^{7}}{\left(a_{i}+a_{j}\right)^{8}} .
\end{aligned}
$$

To our knowledge, analytical expressions for $\alpha_{i j}$ analogous to eqs (94) and (95) have never been derived before.

When all the particles in the dispersion are identical, eqs (92) and (93) reduce to

$$
\begin{gathered}
\langle\mathbf{U}\rangle=\mathbf{U}^{(0)}\left[1+\alpha \varphi+O\left(\varphi^{2}\right)\right] \\
\alpha=-\left(2+3 \beta_{23}\right)-\frac{15}{8} \beta_{23} \beta_{25}-\frac{1}{320} \beta_{23}\left(4 \beta_{(-3) 2}\right. \\
\left.+20 \beta_{(-1) 4}-160 \beta_{05}+91 \beta_{27}\right)+\frac{75}{256} \beta_{23} \beta_{25}^{2} .
\end{gathered}
$$

On the other hand, the average settling velocity in a suspension of identical fluid drops can be determined in the same procedure using the connectoralgebra results for $\hat{x}_{11}^{a}, \hat{x}_{12}^{a}, \hat{y}_{11}^{a}$ and $\hat{y}_{12}^{a}$ accurate to $O\left(r^{-7}\right)$ obtained by Geigenmuller and Mazur (1986).
The coefficient $\alpha$ for this case is

$$
\begin{aligned}
\alpha= & -\frac{4+5 \eta^{*}}{1+\eta^{*}}-\frac{\left(2+3 \eta^{*}\right)\left(2+5 \eta^{*}\right)}{8\left(1+\eta^{*}\right)^{2}} \\
& -\frac{24+55 \eta^{*}-144 \eta^{* 2}-27 \eta^{* 3}}{192\left(1+\eta^{*}\right)^{2}\left(4+\eta^{*}\right)} \\
& +\frac{\left(2+3 \eta^{*}\right)\left(2-5 \eta^{*}\right)^{2}}{256\left(1+\eta^{*}\right)^{3}} .
\end{aligned}
$$

In eqs (97) and (98), all terms except the first one are the contribution from the integral involving the correction function $\mathbf{W}$. It can be found that eqs (97) and (98) are equivalent for the limiting cases of a suspension of no-slip particles $\left(\widehat{\beta} \rightarrow \infty, \eta^{*} \rightarrow \infty\right)$ and of perfect-slip bubbles $\left(\hat{\beta} \rightarrow 0, \eta^{*} \rightarrow 0\right)$.

The accuracy of the value of $\alpha$ in eq. (96) depends on the accuracy of $\mathbf{W}(\mathbf{r})$ defined by eq. (87) or $\mathrm{U}_{t}^{*}(\mathbf{r})$ given by eq. (88) being used in the calculation. Table 3 lists the results of $\alpha$ for the limiting cases of $\hat{\beta} \rightarrow \infty$ and $\hat{\beta}=0$ when the mobility functions $\hat{x}_{11}^{a}, \hat{x}_{12}^{a}, \hat{y}_{11}^{a}$ and $\hat{y}_{12}^{a}$ in eq. (88) are calculated to various accuracies of order of $r^{-1}$ from $O\left(r^{-3}\right)$ to $O\left(r^{-11}\right)$ using eqs (94) and (95). All the previous calculations of $\alpha$ for a suspension of identical solid or fluid spheres in these limits using numerical solutions for the mobility functions are also given in this table for comparison. It can be seen that the convergence of our results of $\alpha$ is not perfect yet even if the accuracy of $O\left(r^{-11}\right)$ is achieved; nevertheless, the agreement between these results and those predicted by Batchelor (1972), Reed and Anderson (1980) and Keh and Tseng (1992) is quite good. 
Table 3. The results of coefficient $\alpha$ defined by eq. (96) for a suspension of identical spheres for the limiting cases of no $\operatorname{slip}(\hat{\beta} \rightarrow \infty)$ and perfect slip $(\hat{\beta}=0)$

\begin{tabular}{ccc}
\hline \multirow{2}{*}{$\begin{array}{c}\text { Accuracy of the } \\
\text { mobility functions } \\
\text { used in eq. (88) }\end{array}$} & \multicolumn{2}{c}{$-\alpha$} \\
\cline { 2 - 3 } & $\hat{\beta} \rightarrow \infty$ & $\hat{\beta}=0$ \\
\hline$O\left(r^{-3}\right)$ & 5.000 & 4.000 \\
$O\left(r^{-4}\right)$ & 6.875 & 4.500 \\
$O\left(r^{-5}\right)$ & 6.875 & 4.500 \\
$O\left(r^{-6}\right)$ & 6.734 & 4.531 \\
$O\left(r^{-7}\right)$ & 6.441 & 4.500 \\
$O\left(r^{-8}\right)$ & 6.391 & 4.504 \\
$O\left(r^{-9}\right)$ & 6.411 & 4.488 \\
$O\left(r^{-10}\right)$ & 6.514 & 4.493 \\
$O\left(r^{-11}\right)$ & 6.553 & 4.486 \\
Batchelor (1972) & 6.55 & - \\
Reed and Anderson (1980) & 6.53 & 4.54 \\
Keh and Tseng (1992) & 6.49 & 4.44 \\
\hline
\end{tabular}

\section{CONCLUDING REMARKS}

In this work, an analytical study for the slow motion of two slip spheres in an infinite fluid is presented using a method of twin multipole expansions. The spheres may have different radii and arbitrary translational and rotational velocities (or applied forces and couples). The resistance and mobility functions that relate the forces and couples to the translational and rotational velocities have been derived in Sections 4 and 5 in the form of power series of $s^{-1}$, where $s$ is the dimensionless distance between the centers of the spheres defined by eq. (8a). The presented results include the hydrodynamic interactions between two no-slip spheres and between two perfectslip spheres (spherical gas bubbles) as special cases. It is found that the particle interaction effects decrease with the increase of the slip coefficients at the particle surfaces. Ying and Peters (1989) also used the twin multipole expansion method to treat the problem of the fluid dynamic interactions of two spheres in a slightly rarefied gas. In their analysis, the boundary condition applied at the particle surfaces was determined from a perturbation expansion of the linearized Boltzmann transport equation for the gas molecules. Their results for the resistance and mobility matrices of the two-sphere system were obtained at small but finite Knudsen numbers and shown to recover Jeffrey and Onishi's (1984) solution when the Knudsen number is zero.

Our solution for the interactions between pairs of spheres has also been utilized to calculate the mean settling velocity in a bounded dispersion of slip spheres. An analytical expression of this mean velocity in the general case is given by eqs (92) and (93). For the limiting cases of no slip and perfect slip at the particle surfaces, our results, expressed by eqs (94) and (95) respectively, are found to agree well with the numerical solutions available in the literature. The mean settling velocity is always reduced as the concentration of particles in the suspension is increased. Again, this effect of retardation is less significant if the slip coefficients at the particle surfaces are increased.

The general construction of the resistance and mobility relations for the motion of two rigid spheres involves the forces, torques, and stresslets exerted by the spheres on the fluid, and the translational and angular velocities of the spheres in the ambient velocity field $\mathbf{U}(\mathbf{x})=\mathbf{U}_{0}+\boldsymbol{\Omega} \times \mathbf{x}+\mathbf{E} \cdot \mathbf{x}$, where $\mathbf{E}$ is a constant rate-of-strain dyadic (Kim and Mifflin, 1985; Kim and Karrila, 1991). Thus, the complete (grand) resistance and mobility matrices are $6 \times 6$ matrices of tensors of second, third and fourth ranks. The axisymmetry about the axis through the sphere centers implies that each tensor can be decomposed into an expression involving no more than three scalar functions. Using the consequences of the stresslets and E being symmetric and traceless, the reciprocal theorem and the two-sphere symmetry, it can be shown that there will be another 12 independent scalar functions (in addition to the 10 functions considered in Section 2) to be determined for either a resistance problem or a mobility problem. For the special case of two no-slip spheres, these 12 resistance functions were calculated using the method of twin multipole expansions by Jeffrey (1992). This analysis can be extended without difficulty to the general case of two slip spheres.

\section{Acknowledgement}

This research was supported by the National Science Council of the Republic of China under Grant No. NSC842214-E002-006.

\begin{tabular}{|c|c|}
\hline \multicolumn{2}{|r|}{ NOTATION } \\
\hline$a$ & particle radius, $\mathrm{m}$ \\
\hline $\mathbf{a}, \mathbf{b}, \tilde{\mathbf{b}}, \mathbf{c}$ & mobility tensors defined by eq. (11) \\
\hline $\mathbf{A}, \mathbf{B}, \tilde{\mathbf{B}}, \mathbf{C}$ & resistance tensors defined by eq. (4) \\
\hline C & $\begin{array}{l}\text { number density of particles in suspen- } \\
\text { sion, } \mathrm{m}^{-3}\end{array}$ \\
\hline$e$ & $\begin{array}{l}\text { unit vector pointing from particle } 1 \text { to } \\
\text { particle } 2\end{array}$ \\
\hline $\mathbf{e}_{\alpha}$ & vector in the direction of $r_{\alpha}$ \\
\hline $\mathbf{F}$ & $\begin{array}{l}\text { force exerted by a particle on the fluid, } \\
\mathrm{N}\end{array}$ \\
\hline$g$ & $\begin{array}{l}\text { two-particle radial distribution func- } \\
\text { tion }\end{array}$ \\
\hline I & unit dyadic \\
\hline $\mathbf{i}, \mathbf{j}, \mathbf{e}$ & $\begin{array}{l}\text { unit vectors in rectangular coordinate } \\
\text { system }\end{array}$ \\
\hline$p$ & dynamic pressure, $\mathrm{Nm}^{-2}$ \\
\hline$p_{m n}, q_{m n}, v_{m n}$ & coefficients defined by eq. (18), $\mathrm{m} \mathrm{s}^{-1}$ \\
\hline $\mathbf{r}$ & $\begin{array}{l}\text { vector pointing from particle } 1 \text { to par- } \\
\text { ticle } 2, \mathrm{~m}\end{array}$ \\
\hline$r$ & equal to $|\mathbf{r}|, \mathrm{m}$ \\
\hline$r_{\alpha}, \theta_{\alpha}, \phi$ & $\begin{array}{l}\text { spherical coordinates with respect to } \\
\text { particle } \alpha\end{array}$ \\
\hline$s$ & {$\left[=2 r /\left(a_{1}+a_{2}\right)\right]$} \\
\hline$t$ & $(=a / r)$ \\
\hline $\mathbf{T}$ & $\begin{array}{l}\text { torque exerted by a particle on the } \\
\mathrm{N} \text { m }\end{array}$ \\
\hline
\end{tabular}


u velocity at particle surface, $\mathrm{m} \mathrm{s}^{-1}$

$\mathbf{U}$ translational velocity of a particle, $\mathrm{ms}^{-1}$

$\langle\mathbf{U}\rangle \quad$ ensemble-averaged sedimentation velocity, $\mathrm{m} \mathrm{s}^{-1}$

v fluid velocity field, $\mathrm{ms}^{-1}$

$V \quad$ volume of the suspension of particles, $\mathrm{m}^{3}$

V undisturbed velocity field of fluid, $\mathrm{ms}^{-1}$

W correction function defined by eq. (87), $\mathrm{ms}^{-1}$

$\mathbf{x} \quad$ position vector, $\mathrm{m}$

$\hat{x}_{\alpha \beta}, \hat{y}_{\alpha \beta} \quad$ dimensionless mobility functions de-

fined by eqs (13) and (14)

$\hat{X}_{\alpha \beta}, \hat{Y}_{\alpha \beta} \quad$ dimensionless resistance functions defined by eqs (7) and (9)

$Y_{m n} \quad$ spherical surface harmonics

\section{Greek letters}

$\alpha$

particle interaction coefficient of $O(\varphi)$ in suspension

$\beta \quad$ reciprocal of the slip coefficient at particle surface, $\mathrm{kg} \mathrm{m}^{-2} \mathrm{~s}^{-1}$

$\hat{\beta} \quad(=\beta a / \eta)$

$\beta_{n m} \quad[=(\hat{\beta}+n) /(\hat{\beta}+m)]$

$\eta \quad$ fluid viscosity, $\mathrm{kg} \mathrm{m}^{-1} \mathrm{~s}^{-1}$

$\eta^{*} \quad$ internal-to-external viscosity ratio of a liquid drop

$\lambda \quad\left(=a_{2} / a_{1}\right)$

$\varphi \quad$ volume fraction of particles in suspension

$\chi_{m n}, \psi_{m n}, \quad$ coefficients defined by eq. (19), $\mathrm{ms}^{-1}$

$\omega_{m n}$

$\omega$

$\Omega$

constant vorticity vector of fluid, $\mathrm{s}^{-1}$ angular velocity of a particle, $s^{-1}$

$\begin{array}{ll}\text { Subscripts } & \\ 0 & \text { particle center } \\ 1,2 & \text { particles } 1,2 \\ i, j & \text { particle types } i, j \\ t & \text { test particle } \\ \alpha, \beta & \text { particles } \alpha, \beta\end{array}$

\section{Superscripts}

(0) infinite dilution

$a, b, c \quad$ tensors $\mathbf{a}, \mathbf{b}, \mathbf{c}$

$A, B, C \quad$ tensors A, B, C

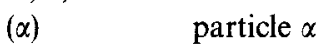

\section{REFERENCES}

Basset, A. B. (1961) A Treatise on Hydrodynamics, Vol. 2. Dover, New York, U.S.A.

Batchelor, G. K. (1972) Sedimentation in a dilute dispersion of spheres. J. Fluid Mech. 52, 245-268.

Batchelor, G. K. (1976) Brownian diffusion of particles with hydrodynamic interaction. J. Fluid Mech. 74, 1-29.

Batchelor, G. K. (1977) The effect of Brownian motion on the bulk stress in a suspension of spherical particles. J. Fluid Mech. 83, 97-117.
Batchelor, G. K. (1982) Sedimentation in a dilute polydisperse system of interacting spheres. Part I, General theory. J. Fluid Mech. 119, 379 408.

Chen, S. H. and Keh, H. J. (1995) Axisymmetric motion of two spherical particles with slip surfaces. J. Colloid Interface Sci. 171, 63-72.

Davis, M. H. (1972) Collisions of small cloud droplets: Gas kinetic effects. J. Atmos. Sci. 29, 911-915.

Felderhof, B. U. (1976) Force density induced on a sphere in linear hydrodynamics. II. Moving sphere, mixed boundary conditions. Physica A 84, $569-576$.

Geigenmuller, U. and Mazur, P. (1986) Many-body hydrodynamic interactions between spherical drops in an emulsion. Physica $A$ 138, 269-298.

Hadamard, J. S. (1911) Mouvement permanent lent d'une sphere liquide et visqueuse dans un liquide visqueux. Compt. Rend. Acad. Sci. (Paris) 152, 1735-1738.

Happel, J, and Brenner, H. (1983) Low Reynolds Number Hydrodynamics. Nijhoff, Dordrecht, The Netherlands.

Hetsroni, G. and Haber, S. (1978) Low Reynolds number motion of two drops submerged in an unbounded arbitrary velocity field. Int. J. Multiphase Flow 4, 1-17.

Hutchins, D. K., Harper, M. H. and Felder, R. L. (1995) Slip correction measurements for solid spherical particles by modulated dynamic light scattering. Aerosol Sci. Technol. 22, 202-218.

Jeffrey, D. J. (1973) Conduction through a random suspension of spheres. Proc. R. Soc. Lond. A 335, $355-367$.

Jeffrey, D. J. (1992) The calculation of the low Reynolds number resistance functions for two unequal spheres. Phys. Fluids A 4, 16-29.

Jeffrey, D. J. and Onishi, Y. (1984) Calculation of the resistance and mobility functions for two unequal rigid spheres in low-Reynolds-number flow. J. Fluid Mech. 139, 261-290.

Keh, H. J. and Tseng, Y. K. (1992) Slow motion of multiple droplets in arbitrary three-dimensional configurations. A.I.Ch.E. J. 38, 1881-1904.

Kennard, E. H. (1938) Kinetic Theory of Gases. McGraw-Hill, New York, U.S.A.

Kim, S. and Karrila, S. J. (1991) Microhydrodynamics: Principles and Selected Applications. ButterworthHeinemann, Boston, MA, U.S.A.

Kim, S. and Mifflin, R. T. (1985) The resistance and mobility functions of two equal spheres in low-Reynolds-number flow. Phys. Fluids 28, 20332045.

Lamb, H. (1945) Hydrodynamics. Dover, New York, U.S.A.

Loyalka, S. K. (1990) Slip and jump coefficients for rarefied gas flows: variational results for Lennard-Jones and $n(r)-6$ potentials. Physica A 163, 813-821.

Reed, C. C. and Anderson, J. L. (1980) Hindered settling of a suspension at low Reynolds number. A.I.Ch.E. J. 26, 816-827.

Reed, L. D. and Morrison, F. A. (1974) Particle interactions in viscous flow at small values of Knudsen number. J. Aerosol Sci. 5, 175-189.

Rybczynski, W. (1911) Uber die fortschreitende bewegung einer flussigen kugel in einem zahen medium. Bull. Acad. Sci. Cracovie Ser. A 1, 40-46. 
Stokes, G. G. (1851) On the effect of the internal friction of fluid on pendulums. Trans. Camb. Phil. Soc. 9, 8-106.

Talbot, L., Cheng, R. K., Scheffer, R. W. and Willis, D. R. (1980) Thermophoresis of particles in a heated boundary layer. J. Fluid Mech. 101, $737-758$
Ying, R. and Peters, M. H. (1989) Hydrodynamic interaction of two unequal-sized spheres in a slightly rarefied gas: resistance and mobility functions. J. Fluid Mech. 207, 353-378.

Ying, R. and Peters, M. H. (1991) Interparticle and particle-surface gas dynamic interactions. Aerosol Sci. Technol. 14, 418-433. 